\title{
Article
}

\section{Comparing a Novel Neuroanimation Experience to Conventional Therapy for High-Dose Intensive Upper-Limb Training in Subacute Stroke: The SMARTS2 Randomized Trial}

Krakauer, John W., Kitago, Tomoko, Goldsmith, Jeff, Ahmad, Omar, Roy, Promit, Stein, Joel, Bishop, Lauri, Casey, Kelly, Valladares, Belen, Harran, Michelle D., Cortés, Juan Camilo, Forrence, Alexander, Xu, Jing, DeLuzio, Sandra, Held, Jeremia P., Schwarz, Anne, Steiner, Levke, Widmer, Mario, Jordan, Kelly, Ludwig, Daniel, Moore, Meghan, Barbera, Marlena, Vora, Isha, Stockley, Rachel, Celnik, Pablo, Zeiler, Steven, Branscheidt, Meret, Kwakkel, Gert and Luft, Andreas R.

Available at http://clok.uclan.ac.uk/37298/

Krakauer, John W., Kitago, Tomoko, Goldsmith, Jeff, Ahmad, Omar, Roy, Promit, Stein, Joel, Bishop, Lauri, Casey, Kelly, Valladares, Belen et al (2021) Comparing a Novel Neuroanimation Experience to Conventional Therapy for High-Dose Intensive Upper-Limb Training in Subacute Stroke: The SMARTS2 Randomized Trial. Neurorehabilitation and Neural Repair, 35 (5). pp. 393-405. ISSN 1545-9683

It is advisable to refer to the publisher's version if you intend to cite from the work. http://dx.doi.org/10.1177/15459683211000730

For more information about UCLan's research in this area go to http://www.uclan.ac.uk/researchgroups/ and search for <name of research Group>.

For information about Research generally at UCLan please go to http://www.uclan.ac.uk/research/ 


\section{CLoK}

Central Lancashire online Knowledge www.clok.uclan.ac.uk 


\section{Neurorehabilitation \& Neural Repair}

\section{Comparing a novel neuroanimation experience to conventional therapy for high-dose intensive upper-limb training in subacute stroke: The SMARTS2 randomized trial}

\begin{tabular}{|c|c|}
\hline Journal: & Neurorehabilitation and Neural Repair \\
\hline Manuscript ID & NNR-20-0383.R3 \\
\hline Manuscript Type: & Original Research Article \\
\hline $\begin{array}{r}\text { Date Submitted by the } \\
\text { Author: }\end{array}$ & $12-F e b-2021$ \\
\hline Complete List of Authors: & 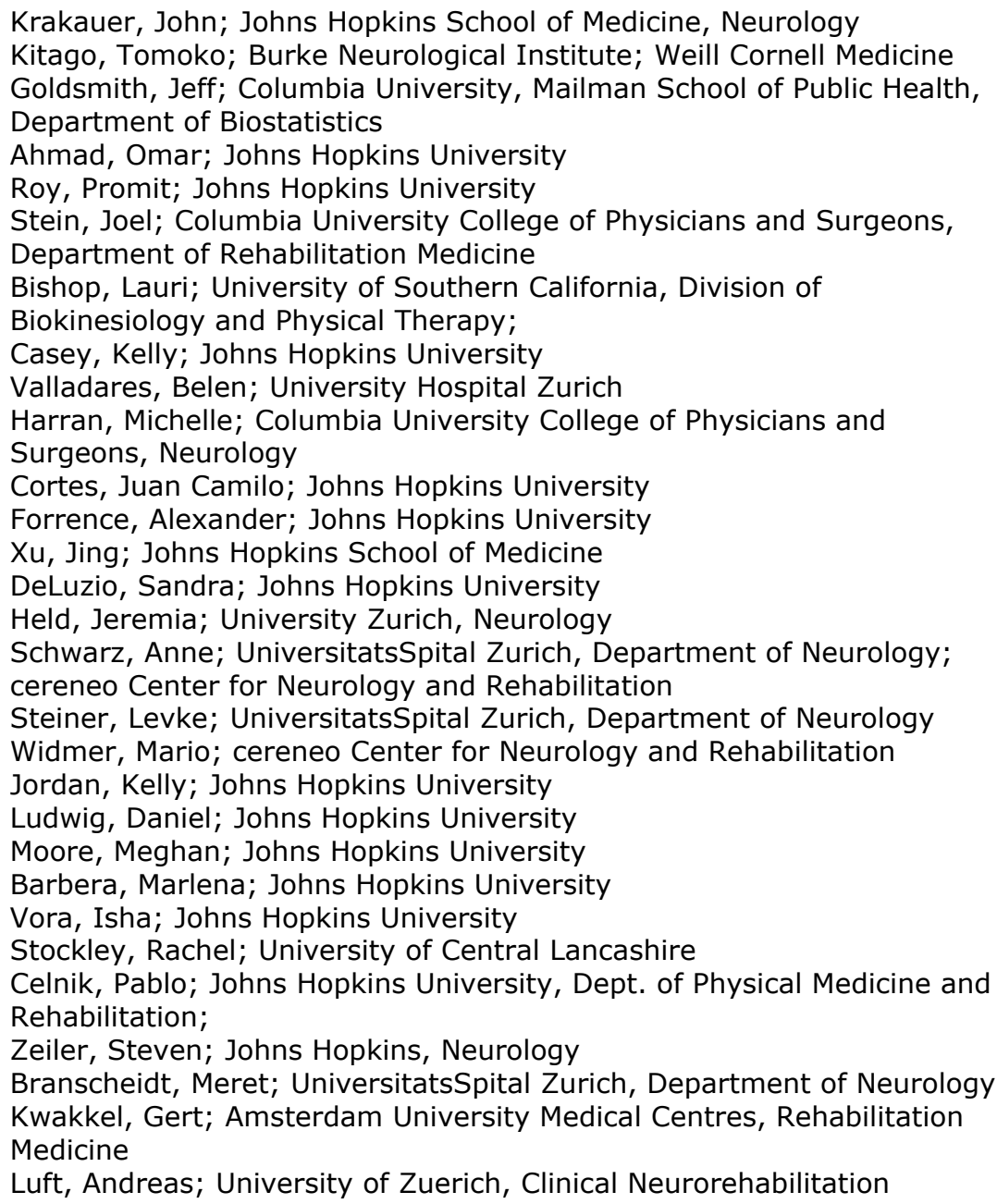 \\
\hline Keyword: & stroke, motor recovery, upper limb, neuroanimation, rehabilitation \\
\hline
\end{tabular}


Background: Evidence from animal studies suggests that greater reductions in post-stroke motor impairment can be attained with significantly higher doses and intensities of therapy focused on movement quality. These studies also indicate a dose-timing interaction, with more pronounced effects if high-intensity therapy is delivered in the acute/subacute, rather than chronic, post-stroke period.

Objective: To compare two approaches of delivering high-intensity, highdose upper limb therapy in patients with subacute stroke: a novel exploratory neuro-animation therapy (NAT), and modified conventional occupational therapy (COT).

Methods: Twenty-four patients were randomized to NAT or COT and underwent 30 sessions of 60 minutes time-on-task in addition to standard care. The primary outcome was the Fugl-Meyer Upper Extremity motor score (FM-UE). Secondary outcomes included: Action Research Arm Test (ARAT), grip strength, Stroke Impact Scale (SIS) hand domain, and upper-limb kinematics. Outcomes were assessed at baseline, and days 3, 90, and 180 post-training. Both groups were compared to a matched historical cohort (HC), which received only 30 minutes of upper limb therapy per day.

Results: There were no significant between-group differences in FM-UE change or any of the secondary outcomes at any timepoint. Both highdose groups showed greater recovery on the ARAT $(7.3 \pm 2.9 \mathrm{pts}$, $p=0.011)$, but not the FM-UE $(1.4 \pm 2.6 \mathrm{pts}, \mathrm{p}=0.564)$ when compared to the $\mathrm{HC}$.

Conclusions: Two forms of high-dose intensive upper limb therapy produced greater activity but not impairment improvements compared with regular care. Neuroanimation may offer a new enjoyable, efficient and scalable way to deliver increased upper limb therapy.

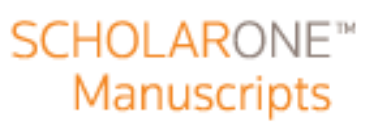




\section{Comparing a novel neuroanimation experience to conventional therapy for high-}

dose intensive upper-limb training in subacute stroke: The SMARTS2 randomized

trial

John W. Krakauer, M.D.1,2,3, Tomoko Kitago, M.D.4,5,6; Jeff Goldsmith, Ph.D.7; Omar Ahmad, Ph.D. ${ }^{1}$; Promit Roy ${ }^{1}$, Joel Stein, M.D.8; Lauri Bishop, Ph.D., D.P.T. ${ }^{8}$, Kelly Casey, O.T.D. ${ }^{3}$, Belen Valladares, M.P.H.9.11, Michelle D. Harran ${ }^{5}$, Juan Camilo Cortés, M.D. ${ }^{1,6}$; Alexander Forrence ${ }^{1}$, Jing Xu, Ph.D. ${ }^{1}$; Sandra DeLuzio ${ }^{3}$, Jeremia P. Held, Ph.D. ${ }^{11}$, Anne Schwarz, M.Sc. ${ }^{11}$, Levke Steiner, M.D. ${ }^{11}$, Mario Widmer, Ph.D. ${ }^{9}$, Kelly Jordan ${ }^{3}$; Daniel Ludwig, D.P.T. ${ }^{3}$, Meghan Moore, D.P.T. ${ }^{3}$, Marlena Barbera ${ }^{3}$, Isha Vora ${ }^{3}$, Rachel Stockley, Ph.D. ${ }^{10}$, Pablo Celnik, M.D. ${ }^{3}$, Steven Zeiler, M.D., Ph.D. ${ }^{1}$; Meret Branscheidt, M.D. ${ }^{11}$, Gert Kwakkel, Ph.D. ${ }^{12,13}$, Andreas R. Luft, M.D.9,11

${ }^{1}$ Dept. of Neurology, Johns Hopkins University, Baltimore, MD, USA

${ }^{2}$ Dept. of Neuroscience, Johns Hopkins University, Baltimore, MD, USA

${ }^{3}$ Dept. of Physical Medicine and Rehabilitation, Johns Hopkins University, Baltimore, USA

${ }^{4}$ Burke Neurological Institute, White Plains, NY, USA

${ }^{5}$ Dept. of Neurology, Weill Cornell Medicine, New York, NY, USA

${ }^{6}$ Dept. of Neurology, Columbia University, New York, NY, USA

${ }^{7}$ Dept. of Biostatistics, Columbia University Mailman School of Public Health, New York, NY, USA

${ }^{8}$ Dept. of Rehabilitation and Regenerative Medicine, Columbia University Vagelos

College of Physicians and Surgeons, New York, USA

${ }^{9}$ cereneo Center for Neurology and Rehabilitation, Vitznau, Switzerland

${ }^{10}$ School of Nursing, University of Central Lancashire, Preston, UK

${ }^{11}$ Division of Vascular Neurology and Neurorehabilitation, Dept. of Neurology,

University Hospital and University of Zurich, Zurich Switzerland

${ }^{12}$ Amsterdam UMC, Vrije Universiteit Amsterdam, Amsterdam, Netherlands

${ }^{13}$ Amsterdam Rehabilitation Research Centre, Reade, Amsterdam, Netherlands

Corresponding author:

John W. Krakauer

Department of Neurology and Neuroscience, Johns Hopkins University

Baltimore, MD 21287

Email: jkrakau1@jhmi.edu

Phone: (410) 955-9320

Abstract word count: 248

Manuscript word count: 6104

Number of figures: 3

Number of tables: 2 


\begin{abstract}
Background: Evidence from animal studies suggests that greater reductions in poststroke motor impairment can be attained with significantly higher doses and intensities of therapy focused on movement quality. These studies also indicate a dose-timing interaction, with more pronounced effects if high-intensity therapy is delivered in the acute/subacute, rather than chronic, post-stroke period.
\end{abstract}

Objective: To compare two approaches of delivering high-intensity, high-dose upper limb therapy in patients with subacute stroke: a novel exploratory neuro-animation therapy (NAT), and modified conventional occupational therapy (COT).

Methods: Twenty-four patients were randomized to NAT or COT and underwent 30 sessions of 60 minutes time-on-task in addition to standard care. The primary outcome was the Fugl-Meyer Upper Extremity motor score (FM-UE). Secondary outcomes included: Action Research Arm Test (ARAT), grip strength, Stroke Impact Scale (SIS) hand domain, and upper-limb kinematics. Outcomes were assessed at baseline, and days 3,90 , and 180 post-training. Both groups were compared to a matched historical cohort $(\mathrm{HC})$, which received only 30 minutes of upper limb therapy per day.

Results: There were no significant between-group differences in FM-UE change or any of the secondary outcomes at any timepoint. Both high-dose groups showed greater 
recovery on the ARAT ( $7.3 \pm 2.9$ pts, $p=0.011)$, but not the FM-UE $(1.4 \pm 2.6 p t s, p$ $=0.564)$ when compared to the HC.

Conclusions: Two forms of high-dose intensive upper limb therapy produced greater activity but not impairment improvements compared with regular care. Neuroanimation may offer a new enjoyable, efficient and scalable way to deliver increased upper limb therapy.

Clinicaltrials.gov registration NCT02292251

Key words: stroke, motor recovery, upper limb, neuroanimation, rehabilitation 


\section{INTRODUCTION}

Current neurorehabilitation approaches in the subacute period after stroke have been ineffective in reducing motor impairment beyond what is expected from spontaneous biological recovery plus standard of care ${ }^{1,2}$. Notably, however, recent studies in patients with chronic stroke have shown improvements with promising effect sizes at both the activity and impairment levels when greatly increased intensities and doses of regular upper limb therapy are provided ${ }^{3-6}$.

A large number of studies in non-human primates (see [8] for extensive review), some going back over a century, have shown that hemiparesis caused by induced focal lesions in motor cortical areas and/or their descending pathways can markedly improve with high-intensity and high-dose training regimens that specifically focus on a return to normal behavior, i.e., movement quality; especially when it is initiated early (within days and weeks of the injury $)^{7,8}$. For example, in one study, monkeys began training on day five post-infarct and were trained on 600 pellet retrievals a day for three to four weeks ${ }^{9}$; full recovery of hand function was seen. In another study, monkeys were trained on food wells with the specific intention that they perform a normal precision grip rather than either of two possible compensatory strategies ${ }^{10}$. This amount and type of upper limb training emphasizing movement quality (normal, non-synergistic movement patterns) of the affected limb is difficult to achieve in the limited amount of time available in standard rehabilitation, which instead by necessity incentivizes task accomplishment via compensation $^{11}$. Furthermore, it has been reported that patients make a total of only about 30 upper limb task-based repetitions during a single therapy session ${ }^{12}$ - almost two orders of magnitude lower than in the cited monkey studies. 
The goals of the SMARTS2 study were the following: 1) to test the feasibility and efficacy of upper limb therapy focused on movement quality when provided at intensities and doses comparable to those given in non-human primate studies, 2) to initiate highintensity and high-dose therapy early ( $<6$ weeks post-stroke), and 3) to compare the efficacy of a new immersive and enjoyable animated experience (neuroanimation therapy, NAT $)^{13}$ versus time-matched conventional occupational therapy (COT). We chose this early time window in the hope of maximizing training benefits, given the extensive evidence in animal models that there is increased responsiveness to training in the first weeks to a month after stroke ${ }^{14}$, and that most spontaneous recovery occurs in the first few months after stroke in humans ${ }^{15}$.

SMARTS2 was a multicenter, single-blinded, parallel randomized controlled trial in subacute stroke, comparing the efficacy of neuroanimation therapy versus intensive conventional occupational therapy. Both types of training provided high-dose, highintensity, movement-quality focused therapy. NAT additionally offered a gamified, motivating environment in which to practice these movements, which we hypothesized might have enhanced efficacy over more traditional forms of therapy. The hypothesis was that NAT would be superior to COT at reducing impairment, as measured with the Fugl-Meyer Upper Extremity motor score (FM-UE), and at least as good at improving arm activity, measured with the Action Research Arm Test (ARAT), as we expected a large reduction in impairment to generalize to activity. The secondary hypothesis was that both forms of high-intensity and high-dose therapy would be better than standardof-care levels of occupational therapy because there is more time to focus on movement quality. 


\section{METHODS}

\section{Study Design}

This was a multicenter, single-blinded, parallel randomized controlled trial comparing the efficacy of a novel exploratory neuroanimation therapy (NAT) with timematched conventional occupational therapy (COT) to enhance upper limb motor recovery after stroke. Eligible patients within six weeks post-stroke were randomized 1:1 to either NAT or COT using a central web-based database (Research Electronic Data Capture, REDCap). Randomization was blocked in groups of six and stratified according to baseline FM-UE score of $6-20$ or 21-40. The randomization sequence was generated by a statistician not involved in the study and concealed from all study team members.

The target therapy schedule for both groups was two daily sessions separated by at least an hour break, five days per week for three weeks, for a total of 30 sessions. Deviations from this schedule were allowed as long as all therapy sessions could be completed within 10 weeks post-stroke. NAT and COT were matched for active therapy time of 60 minutes of time-on-task per session, which was tracked by the gaming software in the NAT group and by stopwatch in the COT group.

Outcome assessments were performed at four timepoints: baseline (pre-training), and post-training day 3 ( \pm 2 days), day 90 ( \pm 10 days), and day 180 ( \pm 10 days). All assessments were conducted by trained evaluators who were blinded to treatment allocation. Patients and caregivers were coached and given a written and verbal reminder not to speak to the evaluator regarding the therapy type at each assessment 
visit. The evaluators had no contact with the participants outside of the assessment sessions to minimize chances of unblinding.

The trial design initially included a third arm consisting of NAT with transcranial direct current stimulation (tDCS). Due to slow initial recruitment, the NAT + tDCS arm was stopped to allow for increased recruitment in the NAT and COT groups, which was our primary comparison of interest. The time window for enrollment post-stroke was also extended from five weeks to six weeks to increase the number of eligible participants.

\section{Study Participants}

Patients were recruited from the acute stroke and inpatient rehabilitation units at Johns Hopkins Hospital, New York Presbyterian Hospital-Columbia, cereneo Center for Neurology and Rehabilitation, and their affiliated institutions. Patients were eligible for the study if they were 21 years old or over, had an ischemic stroke (hemispheric or brainstem) confirmed by CT or MRI within the previous six weeks with residual arm weakness (FM-UE score 6-40 pts), had no history of prior stroke with associated motor deficits, and were able to give informed consent and understand the tasks involved. Exclusion criteria included: intracranial hemorrhage, botulinum toxin injection to upper limb since stroke, physical or neurological condition that interfered with study procedures or assessment of upper limb motor function, inability to sit in a chair and exercise for one hour at a time, participation in another upper limb rehabilitation intervention study, and inability to return for all study sessions. All patients gave written informed consent for participation in the study. The study was approved by the 
institutional review board at each center and registered with Clinicaltrials.gov (NCT02292251) prior to the start of enrollment.

\section{Interventions}

Licensed therapists underwent both in-person and video training on the study interventions at each site. Materials, instructions, and documentation was standardized across sites, and a single therapist (L.B.) supervised training sessions.

Neuroanimation Therapy (NAT). Participants played a custom-designed immersive animation-based experience: I am Dolphin (KATA, Johns Hopkins University) (Figure 1). 3D movements of the paretic arm controlled the movement of a virtual dolphin, swimming through different ocean scenes with various task goals including chasing and eating fish, eluding attacks, and performing jumps. Tasks were designed to promote movement in all planes throughout the active ranges of motion, and titrated based on successful completion of progressive levels of difficulty.

Patient's paretic arm was unweighted using the Armeo®Power (Hocoma AG, Volketswil, Switzerland), an upper limb exoskeleton device. This allowed practice of multijoint 3D arm movements despite antigravity weakness without requiring a therapist to actively lift the paretic arm. The degree of unweighting provided by the exoskeleton was adjusted for each patient to maintain shoulder flexion to 90 degrees at rest so as to provide weight-support of the paretic limb throughout its full active range in all directions. No active assistance was given along the line of movement by the device. The device was integrated with a custom gaming software in a room which simulated an immersive oceanic environment. A large screen displayed the dolphin in his 
environment, oceanic sounds and music were played, and the lights were dimmed for the entirety of each session. A licensed physical or occupational therapist was present throughout each session and provided verbal and tactile feedback to assure high-quality movements (i.e. normal non-synergistic movement patterns) and exploration of the full workspace.

Modified conventional occupational therapy (COT). COT targeting the upper limb was administered by a licensed therapist according to a written standardized protocol. Active COT time was matched to that of the NAT group (60 minutes per session). The Chedoke-McMaster Stroke Assessment Stage ${ }^{16}$ for the hand and arm was administered at the beginning of each week to guide interventions aligned with the specified level of function. Therapeutic exercises consisted of range of motion (stretching) and strengthening exercises of the paretic arm, and training of ADLs, such as simulated cooking/eating, dressing, grooming and cleaning tasks. A typical COT session would include 30 minutes of impairment intervention (e.g. scapular stability, weight bearing, active range of motion, stretching, and strengthening), and 30 minutes of activity training (e.g. reaching, grasping, pinching, bilateral limb coordination during functional activities, ADLs). Therapists were given a list of activities from which to choose but could add additional personalized activities that targeted the specified upper limb movements for the session. Functional activities were broken down into their movement components first and then practiced through gradual progression of complexity and difficulty, similar to shaping strategies employed in constraint-induced movement therapy. Activities were trained using either the paretic or bilateral upper limb(s) as appropriate for successful completion of the task. The first session of the day targeted 
shoulder/elbow, the second session targeted wrist/hand. Therapists focused on movement quality during the COT sessions by preventing compensatory movements and discouraging use of abnormal synergies. The musical soundtrack of NAT was played during COT to maintain blinding if the therapy was conducted in close proximity to blinded evaluators.

All therapeutic activities were documented by the therapy team, including the amount of assistance provided (e.g. passive mobility, active assisted mobility, weight bearing), any modifications to the exercise, and total time spent on each area of the upper limb. Participants were not explicitly instructed with regard to integrating the activities into the home environment.

\section{Clinical study outcomes}

At the baseline visit, patients underwent a comprehensive clinical evaluation that included: medical chart and radiological review, National Institutes of Health Stroke Scale (NIHSS), proprioception assessment (using distal phalanx testing, abnormal defined as $<3 / 5$ trials incorrect), Montreal Cognitive Assessment ${ }^{17}$, Florida Apraxia Battery ${ }^{18}$, star cancellation test of visuospatial neglect (presence of hemineglect determined by cutoff score of $\left.44^{19}\right)$, and Beck Depression Inventory- $\|^{20}$. Study assessments were performed at four timepoints: baseline (pre-training), and posttraining days 3 ( \pm 2 days), 90 ( \pm 10 days), and 180 ( \pm 10 days). All evaluators underwent video and in-person training prior to conducting study assessments and were blinded to patients' treatment allocation. 
The primary outcome measure was the change in upper limb impairment measured by FM-UE, from baseline to post-training day 3. The FM-UE is a widely used, reliable, and validated measure of motor impairment in patients with stroke ${ }^{21}$. It evaluates the ability to make upper limb movements in and out of synergy patterns and consists of 33 items graded on an ordinal scale (0-2), with a best possible score of 66 . The minimal clinically important difference (MCID) for the FM-UE is considered to be approximately $10 \%$ of the maximum score, or 6.6 points ${ }^{21}$.

Secondary clinical outcome measures included changes from baseline to day 3 post-training in: 1) ARAT, an assessment of upper limb activity limitation and dexterity with the MCID for the paretic arm being 12 points ${ }^{22}$ 2) grip strength using a Jamar dynamometer (average of three trials, MCID $5 \mathrm{~kg}^{22}$ ), and 3) the hand domain of the Stroke Impact Scale (SIS hand) version 2.0, a self-reported measure of hand function ${ }^{23}$. All measures were also assessed at 90 and 180 days post-training, to evaluate longerterm gains. Measures of therapy compliance included number of sessions completed and minutes of active therapy within each session.

\author{
Kinematics of planar reaching \\ Motor control of the proximal arm was evaluated using a planar arm reaching \\ task and analyzed using methods that have been previously described ${ }^{15}$ (for detailed \\ information, see Supplemental Materials).
}

Finger strength and individuation 
Finger strength and individuation were evaluated using an ergonomic keyboard device that measures isometric forces produced by each finger ${ }^{24}$ (for detailed information, see Supplemental Materials).

\section{Statistical analysis}

Each outcome measure (FM-UE, ARAT, grip strength, SIS hand, AMD², finger MVF and individuation index) was analyzed using the same framework and model structure. Specifically, we used a linear mixed model in which timepoint was treated as a categorical predictor with four levels (reference: baseline visit), therapy type was a categorical variable with two levels (reference: NAT), and all timepoint by therapy type interactions were included. Additionally, a subject-specific random intercept accounted for within-subject correlation across timepoints. This model structure estimates the mean change in outcome value from baseline to each timepoint as well as the difference in this change over time comparing NAT to COT groups, using all available subject data at each visit. Regression diagnostics were used to assess model fit, and two-sided Wald tests were used to assess statistical significance for group-level comparisons.

We conducted a power analysis for the ability to detect a difference in treatment effect between NAT and COT groups. This analysis was based on a two-sample t-test with two-sided alpha level set at 0.05 and assumed the difference between groups in the change in FM-UE score would be 7 (MCID). For an effect standard deviation of 5 , 10 subjects per group yields $84 \%$ power to detect the true alternative; for an effect standard deviation of 7,20 subjects per group yields $87 \%$ power. We planned to 
randomize 24 subjects in each group, based on our observed attrition rate of $20 \%$ for previous longitudinal stroke studies.

Comparison to historical cohort (HC). In exploratory analyses, we sought to compare FM-UE and ARAT outcomes among patients in the current study, all of whom received an intensive intervention, to patients who received usual clinical care. To do so, we used data from the EXPLICIT trial ${ }^{25}$, which was conducted in the Netherlands and randomized patients within 14 days of first-ever hemiparetic stroke to modified constraint induced movement therapy or usual care. Specifically, patients in the EXPLICIT usual care cohort received occupational therapy based on current Dutch guidelines for 30 minutes a day, five days a week for three weeks beginning within five weeks post-stroke. This amount of therapy approximately matches the average number of minutes spent on therapeutic activities for the upper limb in a typical OT session in the United States ${ }^{12}$. Usual care patients from the EXPLICIT trial were matched to patients in our intense treatment groups (entire SMARTS2 cohort) based on day poststroke ( \pm 4 days) and severity ( \pm 4 points for FM-UE and ARAT). After candidate matches were found, we randomly sampled a single subject to serve as the match for each subject in the intense treatment group. In this analysis, we computed the change in FM-UE and ARAT between baseline and day three post-training for patients in the SMARTS2 group and a similar timeframe for the matched patients in the EXPLICIT usual care group, and compared the average change between groups. Because there can be several potential matches of whom one is selected, we repeated the full analysis and aggregated results to account for uncertainty in the matching process. 


\section{RESULTS}

Between April 2015 and October 2017, 4030 patients with ischemic stroke were assessed for eligibility (Figure 2). The study was stopped after 24 subjects were randomized due to slow recruitment. Table 1 summarizes baseline characteristics of patients who were randomized. The median number of days from stroke onset to baseline assessment was 19.0 days (IQR 12.0, 33.0) and 14.0 (IQR 12.5, 35.5) in the NAT and COT groups, respectively. There were no significant differences in gender, arm affected, proportion receiving tissue plasminogen activator or mechanical thrombectomy, median NIHSS score, proportion with hemineglect, proportion receiving selective serotonin reuptake inhibitors, or baseline FM-UE or ARAT scores. The COT group did have statistically worse scores on the Florida Apraxia Battery, whereas the NAT group had worse depression scores at baseline.

One patient in the NAT group did not receive the intervention due to transportation issues. Two patients withdrew from the study before the end of the planned intervention: one preferred to receive only standard rehabilitation, and one was transferred to another facility. Compliance with active therapy was high in both groups. Excluding the 3 patients who did not receive the intervention or withdrew before the end of therapy, the mean total time in active therapy was 1769 minutes for NAT $(98 \%$ of target) and 1801 for COT (100\% of target). Two patients in the NAT group required minimal assistance from the therapist to initiate movement in the first few sessions but were subsequently able to complete the training on their own. Twenty-one patients 
completed the post-training day 3 assessment (88\%), 20 completed the post-training day 90 assessment (83\%), and 19 completed the day 180 assessment $(79 \%)$.

\section{Clinical outcomes}

Our primary outcome was the change in FM-UE score from baseline (pretraining) to post-training day 3 . There was no significant difference between NAT and COT groups in our primary outcome of FM-UE changes from baseline to day 3 posttraining (difference 1.34, standard error [SE] 5.15, $p=0.797$ ), or in FM-UE changes from baseline to day 90 (difference -3.28 , SE 5.26, $p=0.316$ ) and day 180 (difference -0.09 , SE 5.38, $p=0.132$ ) post-training (Table 2 ). There was similarly no significant difference between NAT and COT for the change in ARAT from baseline to day 3, day 90, or day 180 post-training (Table 2). Grip strength and SIS hand also showed no between-group differences in change from baseline at any timepoint (Table 2).

\section{Reaching kinematics}

We calculated the average squared Mahalanobis distance $\left(A M D^{2}\right)$ for reaching trajectories performed with the paretic arm at the four timepoints, compared to a reference population of neurologically-healthy control subjects. There were 2 patients in the COT group whose reaching kinematic data were excluded from analysis because there were too few movements in the baseline session that were suitable for inclusion in the analysis. An additional 2 patients were missing baseline assessments of reaching kinematics ( 1 in each group) and therefore were not included in the analysis of change scores from baseline. Of the remaining patients, day 90 data were missing for 2 
subjects and day 180 data were missing for 2 patients, due to time constraints or withdrawal from the study. There was improvement in reach kinematics across the intervention period but there was no significant difference in the change in $A M D^{2}$ from baseline between NAT and COT groups at day 3 , day 90 , or day 180 post-training (Table 2). There was a significant correlation between the $\mathrm{AMD}^{2}$ and the ARAT ( $p=$ $0.028)$ but not the FM-UE $(p=0.622)$.

Finger strength and individuation

Maximum voluntary force and finger individuation index were calculated for the more affected arm at the four timepoints as described above. 1 patient in the NAT group did not complete the tasks at baseline and was excluded from analysis. There were also data missing for 4 patients at post-training day 90 ( 2 in NAT and 2 in COT), and 5 patients at post-training day 180 ( 2 in NAT and 3 in COT). We found no significant between-group difference in the change in MVC or individuation index from baseline to any post-training timepoint. (Table 2).

Comparison of intensive therapy to the historical cohort (HC)

In an exploratory analysis, we compared the change in FM-UE and ARAT from baseline to post-training day 3 in our SMARTS2 study cohort (NAT and COT groups combined) to changes across a similar timeframe with usual care in a historical cohort from the EXPLICIT-stroke study ${ }^{25}$. Patients in SMARTS2 were matched by time poststroke and severity (FM-UE and ARAT) with patients from EXPLICIT. We observed a significant benefit in upper limb activity (ARAT difference 7.33, SE 2.88 pts, $p=0.011$ ) 


\begin{abstract}
but not for impairment (FM-UE difference 1.44, SE 2.57, $p=0.564$ ) with the intensive therapy provided in SMARTS2 compared with usual care (Figure 3).
\end{abstract}

\title{
Adverse events
}

There were a total of 55 adverse events (AEs) that occurred in 13 patients during the study. There were 4 serious adverse events in the COT gorup that were unrelated to the study procedures, (2 falls resulting hospitalization and 2 unrelated medical conditions). Of the 51 non-serious AEs, 23 (42\%) occurred in the NAT group and 32 $(58 \%)$ occurred in the COT group. In the NAT group, 5 AEs were probably related (neck pain, fatigue in 3 patients, and bruising) and 6 AEs were possibly related (eye pain in 2 patients, headache, nausea, worsened ataxia, and a fall) to study procedures. In the COT group, two AEs were definitely related (wrist pain in 2 sessions), 1 probably related (pain), and two possibly related (pain, fall) to study procedures.

\section{DISCUSSION}

In this randomized, single-blinded proof-of-concept trial we sought to test the idea, inspired by studies in non-human primates, that high-intensity and high-dose upper limb therapy focused on movement quality rather than task accomplishment, and delivered early after stroke, would reduce motor impairment more than usual care does. We tested this main idea by taking two distinct approaches. The first was administering high doses of conventional upper limb therapy. The second was a new immersive animated experience that centers on a proprietary form of animation designed to promote playful exploration of high quality continuous 3D arm movements ${ }^{13}$. Here we 
found that both approaches led to similar changes in the FM-UE, ARAT, reaching kinematics, finger strength, and finger individuation. Looking at a historical cohort, we found that both approaches were superior to usual care with respect to the ARAT but not the FM-UE. Unfortunately, hand and planar kinematic measures were not available for the historical cohort.

Conventional occupational therapy (COT) mainly emphasizes repetitive taskoriented training (TOT $)^{26,27}$, an approach predicated on practice schedules based on motor learning principles. Not much time is dedicated to the upper limb in regular therapy sessions ${ }^{12}$, which means that TOT focuses more on compensatory movements for task accomplishment. That said, there is nothing inherent to COT that precludes a switch in emphasis to movement quality, especially if therapists are given more time with the patient, as they were in SMARTS2. In three recent studies in patients with chronic stroke, large gains in both the ARAT (or other activity-level measure) and the FM-UE were seen when patients were provided with either five or six hours of upper limb therapy a day for five days/week for three, six or twelve weeks ${ }^{3-5}$. Clearly these are very high intensities and doses of therapy. In two of the studies ${ }^{4,5}$, the authors explicitly state that they wanted to make "movement practice as close to normal as possible", and did so by progressing from single-joint to two-joint movements, then assembling these into task components and finally practicing performance of the full task. It is evident that they combined the more traditional neurophysiological approach, which focuses on movement quality, with TOT. The therapists in our study took a comparable approach in the COT group, as outlined in the methods section. In fact, they explicitly stated on 
questioning that they were able to focus on movement quality precisely because they had more time with the patient.

The NAT and COT groups showed comparable changes in ARAT scores. These changes were significantly greater than the changes seen in the historical control group receiving usual care over the same time period. The ARAT is a valid and responsive measure of upper limb activity on the ICF scale ${ }^{28,29}$ and its components have considerable overlap with the reach and grasp tasks in non-human primate experiments investigating motor recovery. Indeed, the ARAT correlates well with kinematic measures of reach and grasp ${ }^{30}$.

We argue here that the changes in the ARAT in the NAT and COT groups are an indication of true improvement in the quality of arm and hand motor control and not just compensation, even though performance on items scored less than 3 (i.e., "normal") can include compensatory movements. First, we know that the ARAT can show, just like the FM-UE, changes, as we saw here (13.4 and 14.70 points from baseline to day 3 post-training in NAT and COT groups, respectively), that are larger earlier compared to later after stroke ${ }^{31}$. That ARAT changes are greater when high-dose therapy is given earlier than later means suggests that they, at least in part, reflect true restitution and not just learned compensation. Second, if the two intervention groups were just being trained to compensate better than usual care, then this must be because they learned to compensate during the intervention. This would not be possible for the NAT group, however, because there was no functional reach and grasp training. Third, large improvements in activity measures have been seen with intense and high dose COT in patients with chronic stroke ${ }^{5,6}$. These changes seem to be dose-dependent, only 
becoming large when 90 hours or more of treatment are given ${ }^{3-5}$. In contrast, 32 hours does not lead to large ARAT changes ${ }^{31}$. If large ARAT changes were just due to optimizing compensatory strategies it seems unlikely that they would not also be seen after 32 hours of COT. Finally, here we found a significant correlation, as have others ${ }^{30,32}$, between improvement in the quality of arm kinematics and the ARAT. Thus, like in non-human primates ${ }^{8}$, we conclude that intense and high-dose upper limb therapy focused on movement quality can at least partially restore motor control in the upper limb in the subacute period after stroke; it does not just train compensatory movements.

Notably, we did not see an increase in the FM-UE, our primary outcome measure, beyond what was seen in the usual care historical control group. Of course, as we were studying subacute rather than chronic stroke, there were large changes in the FM-UE due to spontaneous recovery but we were not able to augment them with either of our interventions. It is always possible that this is a false negative result given the low $n$, but a previous study of early intense and high-dose upper limb therapy, in this case constraint-induced movement therapy, also reported a dissociation between the ARAT and the FM-UE ${ }^{25}$.

It is possible for ARAT improvements to reflect true changes in motor control and yet not be detected by the FM-UE score due to differences in emphasis for the two scales. For example, a patient who has regained active range of movement in the shoulder/elbow but has persistent difficulty with out of synergy movements (e.g., unable to initiate shoulder flexion or abduction without bending the elbow, which would confer a score of "0" on these FM-UE items) may nevertheless improve on the ARAT by gaining 
the ability to reach the top shelf through improved shoulder flexion and elbow extension. Another situation in which one may see a dissociation between FM-UE and ARAT changes is a patient who primarily regains distal dexterity, which is weighted more heavily on the ARAT than the FM-UE.

The differential responsiveness of the FM-UE and the ARAT to an intervention is not altogether surprising. FM-UE and ARAT measure different constructs of the ICF model, reflecting the levels of body function and activity, respectively. In addition, the FM-UE scale was primarily devised to quantify post-stroke synergies over the course of motor recovery ${ }^{33}$ whereas the ARAT emphasizes assessment of prehension (combined reach and grasp) during more functional tasks ${ }^{29,34}$. In other words, one primarily targets a positive sign and the other a negative sign of the upper motor neuron syndrome ${ }^{8}$. That being said, synergies will intrude on a functional task, especially in the absence of arm weight support ${ }^{35,36}$, which is why the two measures often correlate with each other ${ }^{37,38}$. It is unfortunate that the FM-UE has come to be considered synonymous with overall impairment after stroke, even though it was designed to assess mostly a single component of impairment, namely synergies, over strength or dexterity. This is problematic because thus far it seems that the positive and negative symptoms of stroke respond differently to interventions in the sub-acute period. In this study, we saw significant improvement in ARAT but not in FM-UE with our intensive movement qualityfocused training, and a similar finding was reported when extra sessions of constraintinduced movement therapy were added in the sub-acute period in the EXPLICIT trial ${ }^{25}$. The divergence of ARAT and FM-UE in comparison to usual care was descriptive rather than statistical, due to the challenges in performing a robust statistical comparison 
between values of different scales, the dependence between tests of each outcome, and the repeated matching framework that was used to compare recovery between groups. It is to be hoped that new kinematic measures will soon be developed that can distinguish arm dexterity/quality of motor control from both synergies and compensation during performance of 3D functional tasks ${ }^{39,40}$. This is of utmost importance because hemiparesis in humans appears to be both a deficit disorder related to damage to the corticospinal tract ${ }^{41,42}$ and a movement disorder, perhaps related to upregulation of the reticulospinal tract ${ }^{43-45}$. These positive and negative signs of hemiparesis will likely need distinct forms of intervention.

Our findings for the NAT and COT groups are congruent with what has been reported in many recent neurorehabilitation studies and trials - both the novel intervention group and the control group show similar, and often large, treatment responses. This has been taken as evidence that new technological or pharmacological interventions do not outperform higher intensities and doses of conventional therapy or, by extension, usual clinical care. In a recent review of 15 neurorehabilitation trials conducted in the last five years it is stated: "There is no clear evidence that interventions tested in large multicenter stroke rehabilitation trials are superior to current care. Furthermore, patients benefited from both the experimental and control interventions at both the subacute and chronic stages" 46 . The crucial point being missed here, however, is that control interventions in clinical trials often consist of more, and sometimes considerably more, conventional therapy than is usually given during regular clinical care. This is certainly the case in our study where patients in the COT group received two hours a day of therapy for five consecutive days over three weeks. 
Because of the greatly increased time available, both NAT and COT were able to increase the emphasis on movement quality and discourage use of compensatory movements. At the very least, one can safely say that there was more movementquality-based training than in the historical cohort. In addition, trials tend to select for patients with less comorbidity and fewer cognitive deficits, which allows them to receive higher doses of usual care. Therefore, the positive results for controls in trials do not imply that usual conventional care is equally efficacious to the novel intervention. Indeed, trials that have directly compared higher doses with usual doses of conventional care have found a difference between them ${ }^{47-49}$. Thus, the fact that a new approach, like the NAT here, is not significantly different from high doses of COT should be taken as a reason for optimism. This implies that the new intervention must possess an active ingredient that potentially could be further optimized in terms of efficacy, efficiency, costeffectiveness, and scalability. This situation can be considered analogous to what happened in the evolution of thrombectomy for acute stroke. In 2013 , three trials showed no benefit of thrombectomy over usual care ${ }^{50-52}$. By 2015, five trials showed superiority for thrombectomy ${ }^{53}$. What happened? The two main reasons were choice of the correct technology for clot removal and a change in protocol design.

As with post-stroke thrombolysis, it is possible that we are on the cusp of a change in the delivery and efficacy of upper limb neurorehabilitation. In this case, the correct choice would be to move toward more immersive experiences to promote intense exploratory training with a focus on movement quality. The protocol change would be, as in SMARTS2, to encourage exploratory multi-joint movements outside of a task context for at least two hours a day. Thus, based on the results here, we suggest 
that an alternative to just increasing the amount of time available to administer COT, is to devise technology-based solutions are scalable and that make it easier and more enjoyable to deliver higher doses and intensities of impairment-focused therapy ${ }^{54}$. It should also be emphasized, that even though in this small study we found no significant difference between COT and NAT for delivery of higher doses of intense upper limb therapy, it does not need to be either/or. It may turn out that the two approaches can complement each other. COT could be considered analogous to drills in sport, for example practicing backhands in tennis for an hour. NAT could be the holistic approach where you combine all the components into a full game.

Compliance with therapy was high in both NAT and COT groups, reaching $98 \%$ and $100 \%$ of targeted time on task, respectively. Overall both interventions were safe, with no serious adverse events related to study procedures. There were, however, more adverse events in the COT group than the NAT group. Fatigue was reported more often in the NAT group, which therapists did not always perceive to be a negative because the therapy was designed to be challenging. Other side effects in the NAT group such as transient headache and pain have been reported previously with game-based interventions and are not unexpected with high intensity training ${ }^{55}$.

This study clearly has a number of limitations. First, the number of patients in this proof-of-concept trial was low. Indeed, we recruited only half the number of patients we anticipated. This is attributable both to our inclusion/exclusion criteria and the challenges of providing two hours of time-on-task upper limb therapy, five days a week beginning in the first six weeks after stroke in addition to usual care ${ }^{56,57}$. Furthermore, incorporation of mechanical thrombectomy as standard of care led to fewer patients with 
severe to moderate motor deficits. Second, we were only able to begin our interventions after patients were discharged from in-patient acute rehabilitation at the sites in the United States due to the challenges of delivering high doses of therapy in addition to standard care in the inpatient rehabilitation setting as well as the need for short length of stay ${ }^{58}$; the average start time was therefore about three weeks post-stroke. From our previous work, we have shown that the time window of heightened neuroplasticity responsible for spontaneous recovery, and perhaps for enhanced training-related improvement that takes advantage of this heightened neuroplasticity, might be as short as 5 weeks ${ }^{15}$. This difficulty with our enrollment time window attests to the continuing challenge of conducting neurorehabilitation trials in this early time period after stroke ${ }^{46,58,59}$. Third, we did not track amount of upper limb activity at home during this study, thus are unable to quantify whether either of the training interventions influenced amount of limb use in the real world. Fourth, we had to use a historical usual care group, albeit an extensive and well-matched one ${ }^{25}$. This was necessary because we were not able to ask patients to enroll in a trial offering three weeks of extra care with the chance that they would end up in the control group that got no extra therapy but would nevertheless require them to make trips to the hospital for assessment. Given our strict time window offering the active intervention later was not an option. Another point, as we made above, is that in clinical trials, the control intervention is most often not "usual" care but an amplified and often unrealistic version of it ${ }^{46}$. Here we were fortunate that a cohort existed that delivered care of the upper limb that was close to what patients actually receive in the subacute post-stroke period. 


\section{CONCLUSIONS}

Increasing the dose and intensity of upper limb rehabilitation training early after stroke, with focus on movement quality, led to gains beyond those seen with usual care. This additional improvement was achieved either by having therapists provide much more COT or with a novel exploratory animation-based approach with exoskeletal weight support. This is exciting, as it suggests that an immersive animation-based experience combined with weight-support might pave the way forward for providing high doses of upper limb rehabilitation focused on movement quality in a more efficient, enjoyable, and scalable way at any time post-stroke.

\section{ACKNOWLEDGMENTS}

This study was principally funded by the James S. McDonnell Foundation Grant 220020220 (J.W.K.). Additional funding came from the P\&K Pühringer Foundation (A.R.L.), Sheikh Khalifa Stroke Institute (SKSI) (J.W.K., O.A., P.R., R.S.) and the Neurology and PMR Departments at the Johns Hopkins University School of Medicine. J.G.'s work was supported in part by NIH grant R01NS097423. The EXPLICIT-stroke consortium and the EXPLICIT-stroke cohort were funded by The Netherlands Organization for Health Research and Development (ZonMw Grant No. 89000001). JWK, OA, and PR are co-inventors of the underlying dolphin technology, which has now been licensed to MSquare Healthcare (a MindMaze Inc. Company) and is available as an approved device called MindPod Dolphin. 


\section{REFERENCES}

1. Prabhakaran S, Zarahn E, Riley C, et al. Inter-individual variability in the capacity for motor recovery after ischemic stroke. Neurorehabil Neural Repair. 2008;22(1):64-71.

2. Byblow WD, Stinear CM, Barber PA, Petoe MA, Ackerley SJ. Proportional recovery after stroke depends on corticomotor integrity. Ann Neurol. 2015;78(6):848-859.

3. Ward NS, Brander F, Kelly K. Intensive upper limb neurorehabilitation in chronic stroke: outcomes from the Queen Square programme. J Neurol Neurosurg Psychiatry. 2019;90(5):498-506.

4. Daly JJ, McCabe JP, Holcomb J, Monkiewicz M, Gansen J, Pundik S. Long-Dose Intensive Therapy Is Necessary for Strong, Clinically Significant, Upper Limb Functional Gains and Retained Gains in Severe/Moderate Chronic Stroke. Neurorehabil Neural Repair. 2019;33(7):523-537.

5. McCabe J, Monkiewicz M, Holcomb J, Pundik S, Daly JJ. Comparison of robotics, functional electrical stimulation, and motor learning methods for treatment of persistent upper extremity dysfunction after stroke: a randomized controlled trial. Arch Phys Med Rehabil. 2015;96(6):981-990.

6. Mawase F, Cherry-Allen KM, Xu J, Anaya M, Uehara S, Celnik P. Pushing the rehabiltiation boundaries: hand motor impairment can be reduced in chronic stroke Neurorehabil Neural Repair. 2020; In press.

7. Darling WG, Pizzimenti MA, Morecraft RJ. Functional recovery following motor cortex lesions in non-human primates: experimental implications for human stroke patients. $J$ Integr Neurosci. 2011;10(3):353-384.

8. Krakauer JW, Carmichael ST. Broken Movement: The Neurobiology of Motor Recovery After STroke. Cambridge, MA: The MIT Press; 2017.

9. Nudo RJ, Wise BM, SiFuentes F, Milliken GW. Neural substrates for the effects of rehabilitative training on motor recovery after ischemic infarct. Science. 1996;272:17911794.

10. Murata $\mathrm{Y}$, Higo N, Oishi T, et al. Effects of motor training on the recovery of manual dexterity after primary motor cortex lesion in macaque monkeys. $J$ Neurophysiol. 2008;99(2):773-786.

11. Dobkin $\mathrm{BH}$. Clinical practice. Rehabilitation after stroke. N Engl J Med. 2005;352(16):1677-1684.

12. Lang CE, Macdonald JR, Reisman DS, et al. Observation of amounts of movement practice provided during stroke rehabilitation. Arch Phys Med Rehabil. 2009;90(10):1692-1698.

13. Krakauer JW, Cortes JC. A non-task-oriented approach based on high-dose playful movement exploration for rehabilitation of the upper limb early after stroke: A proposal. NeuroRehabilitation. 2018;43(1):31-40.

14. Biernaskie J, Chernenko G, Corbett D. Efficacy of rehabilitative experience declines with time after focal ischemic brain injury. J Neurosci. 2004;24(5):1245-1254.

15. Cortes JC, Goldsmith J, Harran MD, et al. A Short and Distinct Time Window for Recovery of Arm Motor Control Early After Stroke Revealed With a Global Measure of Trajectory Kinematics. Neurorehabil Neural Repair. 2017;31(6):552-560.

16. Gowland C, Stratford P, Ward M, et al. Measuring physical impairment and disability with the Chedoke-McMaster Stroke Assessment. Stroke. 1993;24(1):58-63.

17. Nasreddine ZS, Phillips NA, Bedirian V, et al. The Montreal Cognitive Assessment, MoCA: a brief screening tool for mild cognitive impairment. J Am Geriatr Soc. 2005;53(4):695-699. 
18. Rothi LJG, Raymer AM, Heilman KM. Apraxia: The neuropsychology of action. . Hove, U.K.: Psychology Press; 1997.

19. Halligan $P$, Wilson $B$, Cockburn J. A short screening test for visual neglect in stroke patients. Int Disabil Stud. 1990;12(3):95-99.

20. Beck AT, Steer RA, Brown GK. Manual for the Beck Depression Inventory-II. San Antonio, TX: Psychological Corporation 1996.

21. Gladstone DJ, Danells CJ, Black SE. The fugl-meyer assessment of motor recovery after stroke: a critical review of its measurement properties. Neurorehabil Neural Repair. 2002;16(3):232-240.

22. Lang CE, Edwards DF, Birkenmeier RL, Dromerick AW. Estimating minimal clinically important differences of upper-extremity measures early after stroke. Arch Phys Med Rehabil. 2008;89(9):1693-1700.

23. Lai SM, Studenski S, Duncan PW, Perera S. Persisting consequences of stroke measured by the Stroke Impact Scale. Stroke. 2002;33(7):1840-1844.

24. Xu J, Ejaz N, Hertler B, et al. Separable systems for recovery of finger strength and control after stroke. J Neurophysiol. 2017;118(2):1151-1163.

25. Kwakkel G, Winters C, van Wegen EE, et al. Effects of Unilateral Upper Limb Training in Two Distinct Prognostic Groups Early After Stroke: The EXPLICIT-Stroke Randomized Clinical Trial. Neurorehabil Neural Repair. 2016;30(9):804-816.

26. Winstein CJ, Wolf SL, Dromerick AW, et al. Effect of a Task-Oriented Rehabilitation Program on Upper Extremity Recovery Following Motor Stroke: The ICARE Randomized Clinical Trial. Jama. 2016;315(6):571-581.

27. Winstein CJ, Stein J, Arena R, et al. Guidelines for Adult Stroke Rehabilitation and Recovery: A Guideline for Healthcare Professionals From the American Heart Association/American Stroke Association. Stroke. 2016;47(6):e98-e169.

28. van der Lee JH, Beckerman H, Lankhorst GJ, Bouter LM. The responsiveness of the action research arm test and the Fugl-Meyer Assessment scale in chronic stroke patients. Journal of Rehabilitation Medicine. 2001;33(3):110-113.

29. Yozbatiran N, Der-Yeghiaian L, Cramer SC. A standardized approach to performing the action research arm test. Neurorehabil Neural Repair. 2008;22(1):78-90.

30. Lang CE, Wagner JM, Dromerick AW, Edwards DF. Measurement of upper-extremity function early after stroke: properties of the action research arm test. Arch Phys Med Rehabil. 2006;87(12):1605-1610.

31. Lang CE, Strube MJ, Bland MD, et al. Dose response of task-specific upper limb training in people at least 6 months poststroke: A phase II, single-blind, randomized, controlled trial. Ann Neurol. 2016;80(3):342-354.

32. Alt Murphy M, Willen C, Sunnerhagen KS. Responsiveness of upper extremity kinematic measures and clinical improvement during the first three months after stroke.

Neurorehabil Neural Repair. 2013;27(9):844-853.

33. Fugl-Meyer AR, Jaasko L, Leyman I, Olsson S, Steglind S. The post-stroke hemiplegic patient. 1. a method for evaluation of physical performance. Scand J Rehabil Med. 1975;7(1):13-31.

34. Lyle RC. A performance test for assessment of upper limb function in physical rehabilitation treatment and research. Int J Rehabil Res. 1981;4(4):483-492.

35. Zackowski KM, Dromerick AW, Sahrmann SA, Thach WT, Bastian AJ. How do strength, sensation, spasticity and joint individuation relate to the reaching deficits of people with chronic hemiparesis? Brain. 2004;127(Pt 5):1035-1046.

36. Lan Y, Yao J, Dewald JPA. The Impact of Shoulder Abduction Loading on Volitional Hand Opening and Grasping in Chronic Hemiparetic Stroke. Neurorehabil Neural Repair. 2017;31(6):521-529. 
37. Platz T, Pinkowski C, van Wijck F, Kim IH, di Bella P, Johnson G. Reliability and validity of arm function assessment with standardized guidelines for the Fugl-Meyer Test, Action Research Arm Test and Box and Block Test: a multicentre study. Clin Rehabil. 2005;19(4):404-411.

38. Wei XJ, Tong KY, Hu XL. The responsiveness and correlation between Fugl-Meyer Assessment, Motor Status Scale, and the Action Research Arm Test in chronic stroke with upper-extremity rehabilitation robotic training. Int J Rehabil Res. 2011;34(4):349356.

39. Kwakkel G, Van Wegen E, Burridge JH, et al. Standardized measurement of quality of upper limb movement after stroke: Consensus-based core recommendations from the Second Stroke Recovery and Rehabilitation Roundtable. Int J Stroke. 2019;14(8):783791.

40. Schwarz A, Kanzler CM, Lambercy O, Luft AR, Veerbeek JM. Systematic Review on Kinematic Assessments of Upper Limb Movements After Stroke. Stroke. 2019;50(3):718-727.

41. Tower SS. Pyramidal lesion in the monkey. Brain. 1940;63(1):36-90.

42. Beebe JA, Lang CE. Absence of a proximal to distal gradient of motor deficits in the upper extremity early after stroke. Clin Neurophysiol. 2008;119(9):2074-2085.

43. Owen M, Ingo C, Dewald JPA. Upper Extremity Motor Impairments and Microstructural Changes in Bulbospinal Pathways in Chronic Hemiparetic Stroke. Front Neurol. 2017;8:257.

44. Ellis MD, Drogos J, Carmona C, Keller T, Dewald JP. Neck rotation modulates flexion synergy torques, indicating an ipsilateral reticulospinal source for impairment in stroke. $J$ Neurophysiol. 2012;108(11):3096-3104.

45. Zaaimi B, Edgley SA, Soteropoulos DS, Baker SN. Changes in descending motor pathway connectivity after corticospinal tract lesion in macaque monkey. Brain. 2012;135(Pt 7):2277-2289.

46. Stinear CM, Lang CE, Zeiler S, Byblow WD. Advances and challenges in stroke rehabilitation. Lancet Neurol. 2020;19(4):348-360.

47. Lo AC, Guarino PD, Richards LG, et al. Robot-assisted therapy for long-term upper-limb impairment after stroke. N Engl J Med. 2010;362(19):1772-1783.

48. Rodgers $\mathrm{H}$, Bosomworth $\mathrm{H}$, Krebs $\mathrm{HI}$, et al. Robot assisted training for the upper limb after stroke (RATULS): a multicentre randomised controlled trial. Lancet. 2019;394(10192):51-62.

49. Wolf SL, Winstein CJ, Miller JP, et al. Effect of constraint-induced movement therapy on upper extremity function 3 to 9 months after stroke: the EXCITE randomized clinical trial. Jama. 2006;296(17):2095-2104.

50. Kidwell CS, Jahan R, Gornbein J, et al. A trial of imaging selection and endovascular treatment for ischemic stroke. N Engl J Med. 2013;368(10):914-923.

51. Broderick JP, Palesch YY, Demchuk AM, et al. Endovascular therapy after intravenous tPA versus t-PA alone for stroke. N Engl J Med. 2013;368(10):893-903.

52. Ciccone A, Valvassori L, Nichelatti M, et al. Endovascular treatment for acute ischemic stroke. N Engl J Med. 2013;368(10):904-913.

53. Moore JM, Griessenauer CJ, Gupta R, et al. Landmark papers in cerebrovascular neurosurgery 2015. Clin Neurol Neurosurg. 2016;148:22-28.

54. Wuennemann MJ, Mackenzie SW, Lane HP, et al. Dose and staffing comparison study of upper limb device-assisted therapy. NeuroRehabilitation. 2020.

55. Laver KE, Lange B, George S, Deutsch JE, Saposnik G, Crotty M. Virtual reality for stroke rehabilitation. Cochrane Database Syst Rev. 2017;11:CD008349. 
56. Cramer SC, Wolf SL, Adams HP, Jr., et al. Stroke Recovery and Rehabilitation Research: Issues, Opportunities, and the National Institutes of Health StrokeNet. Stroke. 2017;48(3):813-819.

57. Stinear CM. Stroke rehabilitation research needs to be different to make a difference. F1000Res. 2016;5.

58. Raghavan P. Research in the Acute Rehabilitation Setting: a Bridge Too Far? Curr Neurol Neurosci Rep. 2019;19(1):4.

59. Stinear C, Ackerley S, Byblow W. Rehabilitation is initiated early after stroke, but most motor rehabilitation trials are not: a systematic review. Stroke; a journal of cerebral circulation. 2013;44(7):2039-2045. 


\title{
Comparing a novel neuroanimation experience to conventional therapy for high-
}

\author{
dose $_{\bar{y}}$ intensive upper-limb training in subacute stroke: The SMARTS2
}

\section{randomized trial}

John W. Krakauer, M.D.1,2,3, Tomoko Kitago, M.D.4,5,6; Jeff Goldsmith, Ph.D.7; Omar Ahmad, Ph.D. ${ }^{1}$; Promit Roy ${ }^{1}$, Joel Stein, M.D.8; Lauri Bishop, Ph.D., D.P.T. ${ }^{8}$, Kelly Casey, O.T.D. ${ }^{3}$, Belen Valladares, M.P.H.9.11, Michelle D. Harran ${ }^{5}$, Juan Camilo Cortés, M.D. ${ }^{1,6}$; Alexander Forrence ${ }^{1}$, Jing Xu, Ph.D. ${ }^{1}$; Sandra DeLuzio ${ }^{3}$, Jeremia P. Held, Ph.D. ${ }^{11}$, Anne Schwarz, M.Sc. ${ }^{11}$, Levke Steiner, M.D. ${ }^{11}$, Mario Widmer, Ph.D. ${ }^{9}$, Kelly Jordan ${ }^{3}$; Daniel Ludwig, D.P.T. ${ }^{3}$, Meghan Moore, D.P.T. ${ }^{3}$, Marlena Barbera ${ }^{3}$, Isha Vora ${ }^{3}$, Rachel Stockley, Ph.D. ${ }^{10}$, Pablo Celnik, M.D. ${ }^{3}$, Steven Zeiler, M.D., Ph.D. ${ }^{1}$; Meret Branscheidt, M.D. ${ }^{11}$, Gert Kwakkel, Ph.D. ${ }^{12,13}$, Andreas R. Luft, M.D.9,11

${ }^{1}$ Dept. of Neurology, Johns Hopkins University, Baltimore, MD, USA

${ }^{2}$ Dept. of Neuroscience, Johns Hopkins University, Baltimore, MD, USA

${ }^{3}$ Dept. of Physical Medicine and Rehabilitation, Johns Hopkins University, Baltimore, USA

${ }^{4}$ Burke Neurological Institute, White Plains, NY, USA

${ }^{5}$ Dept. of Neurology, Weill Cornell Medicine, New York, NY, USA

${ }^{6}$ Dept. of Neurology, Columbia University, New York, NY, USA

${ }^{7}$ Dept. of Biostatistics, Columbia University Mailman School of Public Health, New York, NY, USA

${ }^{8}$ Dept. of Rehabilitation and Regenerative Medicine, Columbia University Vagelos

College of Physicians and Surgeons, New York, USA

${ }^{9}$ cereneo Center for Neurology and Rehabilitation, Vitznau, Switzerland

${ }^{10}$ School of Nursing, University of Central Lancashire, Preston, UK

${ }^{11}$ Division of Vascular Neurology and Neurorehabilitation, Dept. of Neurology,

University Hospital and University of Zurich, Zurich Switzerland

${ }^{12}$ Amsterdam UMC, Vrije Universiteit Amsterdam, Amsterdam, Netherlands

${ }^{13}$ Amsterdam Rehabilitation Research Centre, Reade, Amsterdam, Netherlands

Corresponding author:

John W. Krakauer

Department of Neurology and Neuroscience, Johns Hopkins University

Baltimore, MD 21287

Email: jkrakau1@jhmi.edu

Phone: (410) 955-9320

Abstract word count: 248

Manuscript word count: 6127ㅈ104

Number of figures: 3

Number of tables: 2 


\begin{abstract}
Background: Evidence from animal studies suggests that greater reductions in poststroke motor impairment can be attained with significantly higher doses and intensities of therapy focused on movement quality. These studies also indicate a dose-timing interaction, with more pronounced effects if high-intensity therapy is delivered in the acute/subacute, rather than chronic, post-stroke period.
\end{abstract}

Objective: To compare two approaches of delivering high-intensity, high-dose upper limb therapy in patients with subacute stroke: a novel exploratory neuro-animation therapy (NAT), and modified conventional occupational therapy (COT).

Methods: Twenty-four patients were randomized to NAT or COT and underwent 30 sessions of 60 minutes time-on-task in addition to standard care. The primary outcome was the Fugl-Meyer Upper Extremity motor score (FM-UE). Secondary outcomes included: Action Research Arm Test (ARAT), grip strength, Stroke Impact Scale (SIS) hand domain, and upper-limb kinematics. Outcomes were assessed at baseline, and days 3,90 , and 180 post-training. Both groups were compared to a matched historical cohort $(\mathrm{HC})$, which received only 30 minutes of upper limb therapy per day.

Results: There were no significant between-group differences in FM-UE change or any of the secondary outcomes at any timepoint. Both high-dose groups showed greater 
recovery on the ARAT ( $7.3 \pm 2.9$ pts, $p=0.011)$, but not the FM-UE $(1.4 \pm 2.6 p t s, p$ $=0.564)$ when compared to the HC.

Conclusions: Two forms of high-dose intensive upper limb therapy produced greater activity but not impairment improvements compared with regular care. Neuroanimation may offer a new enjoyable, efficient and scalable way to deliver increased upper limb therapy.

Clinicaltrials.gov registration NCT02292251

Key words: stroke, motor recovery, upper limb, neuroanimation, rehabilitation 


\section{INTRODUCTION}

Current neurorehabilitation approaches in the subacute period after stroke have been ineffective in reducing motor impairment beyond what is expected from spontaneous biological recovery plus standard of care ${ }^{1,2}$. Notably, however, recent studies in patients with chronic stroke have shown improvements with promising effect sizes at both the activity and impairment levels when greatly increased intensities and doses of regular upper limb therapy are provided ${ }^{3-6}$.

A large number of studies in non-human primates (see [8] for extensive review), some going back over a century, have shown that hemiparesis caused by induced focal lesions in motor cortical areas and/or their descending pathways can markedly improve with high-intensity and high-dose training regimens that specifically focus on a return to normal behavior, i.e., movement quality; especially when it is initiated early (within days and weeks of the injury $)^{7,8}$. For example, in one study, monkeys began training on day five post-infarct and were trained on 600 pellet retrievals a day for three to four weeks ${ }^{9}$; full recovery of hand function was seen. In another study, monkeys were trained on food wells with the specific intention that they perform a normal precision grip rather than either of two possible compensatory strategies ${ }^{10}$. This amount and type of upper limb training emphasizing movement quality (normal, non-synergistic movement patterns) of the affected limb is difficult to achieve in the limited amount of time available in standard rehabilitation, which instead by necessity incentivizes task accomplishment via compensation $^{11}$. Furthermore, it has been reported that patients make a total of only about 30 upper limb task-based repetitions during a single therapy session ${ }^{12}$ - almost two orders of magnitude lower than in the cited monkey studies. 
The goals of the SMARTS2 study were the following: 1) to test the feasibility and efficacy of upper limb therapy focused on movement quality when provided at intensities and doses comparable to those given in non-human primate studies, 2) to initiate highintensity and high-dose therapy early ( $<6$ weeks post-stroke), and 3) to compare the efficacy of a new immersive and enjoyable animated experience (neuroanimation therapy, NAT $)^{13}$ versus time-matched conventional occupational therapy (COT). We chose this early time window in the hope of maximizing training benefits, given the extensive evidence in animal models that there is increased responsiveness to training in the first weeks to a month after stroke ${ }^{14}$, and that most spontaneous recovery occurs in the first few months after stroke in humans ${ }^{15}$.

SMARTS2 was a multicenter, single-blinded, parallel randomized controlled trial in subacute stroke, comparing the efficacy of neuroanimation therapy versus intensive conventional occupational therapy. Both types of training provided high-dose, highintensity, movement-quality focused therapy. NAT additionally offered a gamified, motivating environment in which to practice these movements, which we hypothesized might have enhanced efficacy over more traditional forms of therapy. The hypothesis was that NAT would be superior to COT at reducing impairment, as measured with the Fugl-Meyer Upper Extremity motor score (FM-UE), and at least as good at improving arm activity, measured with the Action Research Arm Test (ARAT), as we expected a large reduction in impairment to generalize to activity. The secondary hypothesis was that both forms of high-intensity and high-dose therapy would be better than standardof-care levels of occupational therapy because there is more time to focus on movement quality. 


\section{METHODS}

\section{Study Design}

This was a multicenter, single-blinded, parallel randomized controlled trial comparing the efficacy of a novel exploratory neuroanimation therapy (NAT) with timematched conventional occupational therapy (COT) to enhance upper limb motor recovery after stroke. Eligible patients within six weeks post-stroke were randomized 1:1 to either NAT or COT using a central web-based database (Research Electronic Data Capture, REDCap). Randomization was blocked in groups of six and stratified according to baseline FM-UE score of 6-20 or 21-40. The randomization sequence was generated by a statistician not involved in the study and concealed from all study team members.

The target therapy schedule for both groups was two daily sessions separated by at least an hour break, five days per week for three weeks, for a total of 30 sessions. Deviations from this schedule were allowed as long as all therapy sessions could be completed within 10 weeks post-stroke. NAT and COT were matched for active therapy time of 60 minutes of time-on-task per session, which was tracked by the gaming software in the NAT group and by stopwatch in the COT group.

Outcome assessments were performed at four timepoints: baseline (pre-training), and post-training day 3 ( \pm 2 days), day 90 ( \pm 10 days), and day 180 ( \pm 10 days). All assessments were conducted by trained evaluators who were blinded to treatment allocation. Patients and caregivers were coached and given a written and verbal reminder not to speak to the evaluator regarding the therapy type at each assessment 
visit. The evaluators had no contact with the participants outside of the assessment sessions to minimize chances of unblinding.

The trial design initially included a third arm consisting of NAT with transcranial direct current stimulation (tDCS). Due to slow initial recruitment, the NAT + tDCS arm was stopped to allow for increased recruitment in the NAT and COT groups, which was our primary comparison of interest. The time window for enrollment post-stroke was also extended from five weeks to six weeks to increase the number of eligible participants.

\section{Study Participants}

Patients were recruited from the acute stroke and inpatient rehabilitation units at Johns Hopkins Hospital, New York Presbyterian Hospital-Columbia, cereneo Center for Neurology and Rehabilitation, and their affiliated institutions. Patients were eligible for the study if they were 21 years old or over, had an ischemic stroke (hemispheric or brainstem) confirmed by CT or MRI within the previous six weeks with residual arm weakness (FM-UE score 6-40 pts), had no history of prior stroke with associated motor deficits, and were able to give informed consent and understand the tasks involved. Exclusion criteria included: intracranial hemorrhage, botulinum toxin injection to upper limb since stroke, physical or neurological condition that interfered with study procedures or assessment of upper limb motor function, inability to sit in a chair and exercise for one hour at a time, participation in another upper limb rehabilitation intervention study, and inability to return for all study sessions. All patients gave written informed consent for participation in the study. The study was approved by the 
institutional review board at each center and registered with Clinicaltrials.gov (NCT02292251) prior to the start of enrollment.

\section{Interventions}

Licensed therapists underwent both in-person and video training on the study interventions at each site. Materials, instructions, and documentation was standardized across sites, and a single therapist (L.B.) supervised training sessions.

Neuroanimation Therapy (NAT). Participants played a custom-designed immersive animation-based experience: I am Dolphin (KATA, Johns Hopkins University) (Figure 1). 3D movements of the paretic arm controlled the movement of a virtual dolphin, swimming through different ocean scenes with various task goals including chasing and eating fish, eluding attacks, and performing jumps. Tasks were designed to promote movement in all planes throughout the active ranges of motion, and titrated based on successful completion of progressive levels of difficulty.

Patient's paretic arm was unweighted using the Armeo®Power (Hocoma AG, Volketswil, Switzerland), an upper limb exoskeleton device. This allowed practice of multijoint 3D arm movements despite antigravity weakness without requiring a therapist to actively lift the paretic arm. The degree of unweighting provided by the exoskeleton was adjusted for each patient to maintain shoulder flexion to 90 degrees at rest so as to provide weight-support of the paretic limb throughout its full active range in all directions. No active assistance was given along the line of movement by the device. The device was integrated with a custom gaming software in a room which simulated an immersive oceanic environment. A large screen displayed the dolphin in his 
environment, oceanic sounds and music were played, and the lights were dimmed for the entirety of each session. A licensed physical or occupational therapist was present throughout each session and provided verbal and tactile feedback to assure high-quality movements (i.e. normal non-synergistic movement patterns) and exploration of the full workspace.

Modified conventional occupational therapy (COT). COT targeting the upper limb was administered by a licensed therapist according to a written standardized protocol. Active COT time was matched to that of the NAT group (60 minutes per session). The Chedoke-McMaster Stroke Assessment Stage ${ }^{16}$ for the hand and arm was administered at the beginning of each week to guide interventions aligned with the specified level of function. Therapeutic exercises consisted of range of motion (stretching) and strengthening exercises of the paretic arm, and training of ADLs, such as simulated cooking/eating, dressing, grooming and cleaning tasks. A typical COT session would include 30 minutes of impairment intervention (e.g. scapular stability, weight bearing, active range of motion, stretching, and strengthening), and 30 minutes of activity training (e.g. reaching, grasping, pinching, bilateral limb coordination during functional activities, ADLs). Therapists were given a list of activities from which to choose but could add additional personalized activities that targeted the specified upper limb movements for the session. Functional activities were broken down into their movement components first and then practiced through gradual progression of complexity and difficulty, similar to shaping strategies employed in constraint-induced movement therapy. Activities were trained using either the paretic or bilateral upper limb(s) as appropriate for successful completion of the task. The first session of the day targeted 
shoulder/elbow, the second session targeted wrist/hand. Therapists focused on movement quality during the COT sessions by preventing compensatory movements and discouraging use of abnormal synergies. The musical soundtrack of NAT was played during COT to maintain blinding if the therapy was conducted in close proximity to blinded evaluators.

All therapeutic activities were documented by the therapy team, including the amount of assistance provided (e.g. passive mobility, active assisted mobility, weight bearing), any modifications to the exercise, and total time spent on each area of the upper limb. Participants were not explicitly instructed with regard to integrating the activities into the home environment.

\section{Clinical study outcomes}

At the baseline visit, patients underwent a comprehensive clinical evaluation that included: medical chart and radiological review, National Institutes of Health Stroke Scale (NIHSS), proprioception assessment (using distal phalanx testing, abnormal defined as $<3 / 5$ trials incorrect), Montreal Cognitive Assessment ${ }^{17}$, Florida Apraxia Battery ${ }^{18}$, star cancellation test of visuospatial neglect (presence of hemineglect determined by cutoff score of $\left.44^{19}\right)$, and Beck Depression Inventory- $\|^{20}$. Study assessments were performed at four timepoints: baseline (pre-training), and posttraining days 3 ( \pm 2 days), 90 ( \pm 10 days), and 180 ( \pm 10 days). All evaluators underwent video and in-person training prior to conducting study assessments and were blinded to patients' treatment allocation. 
The primary outcome measure was the change in upper limb impairment measured by FM-UE, from baseline to post-training day 3. The FM-UE is a widely used, reliable, and validated measure of motor impairment in patients with stroke ${ }^{21}$. It evaluates the ability to make upper limb movements in and out of synergy patterns and consists of 33 items graded on an ordinal scale (0-2), with a best possible score of 66 . The minimal clinically important difference (MCID) for the FM-UE is considered to be approximately $10 \%$ of the maximum score, or 6.6 points ${ }^{21}$.

Secondary clinical outcome measures included changes from baseline to day 3 post-training in: 1) ARAT, an assessment of upper limb activity limitation and dexterity with the MCID for the paretic arm being 12 points ${ }^{22}$ 2) grip strength using a Jamar dynamometer (average of three trials, MCID $5 \mathrm{~kg}^{22}$ ), and 3) the hand domain of the Stroke Impact Scale (SIS hand) version 2.0, a self-reported measure of hand function ${ }^{23}$. All measures were also assessed at 90 and 180 days post-training, to evaluate longerterm gains. Measures of therapy compliance included number of sessions completed and minutes of active therapy within each session.

\author{
Kinematics of planar reaching \\ Motor control of the proximal arm was evaluated using a planar arm reaching \\ task and analyzed using methods that have been previously described ${ }^{15}$ (for detailed \\ information, see Supplemental Materials).
}

Finger strength and individuation 
Finger strength and individuation were evaluated using an ergonomic keyboard device that measures isometric forces produced by each finger ${ }^{24}$ (for detailed information, see Supplemental Materials).

\section{Statistical analysis}

Each outcome measure (FM-UE, ARAT, grip strength, SIS hand, $A M D^{2}$, finger MVF and individuation index) was analyzed using the same framework and model structure. Specifically, we used a linear mixed model in which timepoint was treated as a categorical predictor with four levels (reference: baseline visit), therapy type was a categorical variable with two levels (reference: NAT), and all timepoint by therapy type interactions were included. Additionally, a subject-specific random intercept accounted for within-subject correlation across timepoints. This model structure estimates the mean change in outcome value from baseline to each timepoint as well as the difference in this change over time comparing NAT to COT groups, using all available subject data at each visit. Regression diagnostics were used to assess model fit, and two-sided Wald tests were used to assess statistical significance for group-level comparisons.

We conducted a power analysis for the ability to detect a difference in treatment effect between NAT and COT groups. This analysis was based on a two-sample t-test with two-sided alpha level set at 0.05 and assumed the difference between groups in the change in FM-UE score would be 7 (MCID). For an effect standard deviation of 5 , 10 subjects per group yields $84 \%$ power to detect the true alternative; for an effect standard deviation of 7,20 subjects per group yields $87 \%$ power. We planned to 
randomize 24 subjects in each group, based on our observed attrition rate of $20 \%$ for previous longitudinal stroke studies.

Comparison to historical cohort (HC). In exploratory analyses, we sought to compare FM-UE and ARAT outcomes among patients in the current study, all of whom received an intensive intervention, to patients who received usual clinical care. To do so, we used data from the EXPLICIT trial ${ }^{25}$, which was conducted in the Netherlands and randomized patients within 14 days of first-ever hemiparetic stroke to modified constraint induced movement therapy or usual care. Specifically, patients in the EXPLICIT usual care cohort received occupational therapy based on current Dutch guidelines for 30 minutes a day, five days a week for three weeks beginning within five weeks post-stroke. This amount of therapy approximately matches the average number of minutes spent on therapeutic activities for the upper limb in a typical OT session in the United States ${ }^{12}$. Usual care patients from the EXPLICIT trial were matched to patients in our intense treatment groups (entire SMARTS2 cohort) based on day poststroke ( \pm 4 days) and severity ( \pm 4 points for FM-UE and ARAT). After candidate matches were found, we randomly sampled a single subject to serve as the match for each subject in the intense treatment group. In this analysis, we computed the change in FM-UE and ARAT between baseline and day three post-training for patients in the SMARTS2 group and a similar timeframe for the matched patients in the EXPLICIT usual care group, and compared the average change between groups. Because there can be several potential matches of whom one is selected, we repeated the full analysis and aggregated results to account for uncertainty in the matching process. 


\section{RESULTS}

Between April 2015 and October 2017, 4030 patients with ischemic stroke were assessed for eligibility (Figure 2). The study was stopped after 24 subjects were randomized due to slow recruitment. Table 1 summarizes baseline characteristics of patients who were randomized. The median number of days from stroke onset to baseline assessment was 19.0 days (IQR 12.0, 33.0) and 14.0 (IQR 12.5, 35.5) in the NAT and COT groups, respectively. There were no significant differences in gender, arm affected, proportion receiving tissue plasminogen activator or mechanical thrombectomy, median NIHSS score, proportion with hemineglect, proportion receiving selective serotonin reuptake inhibitors, or baseline FM-UE or ARAT scores. The COT group did have statistically worse scores on the Florida Apraxia Battery, whereas the NAT group had worse depression scores at baseline.

One patient in the NAT group did not receive the intervention due to transportation issues. Two patients withdrew from the study before the end of the planned intervention: one preferred to receive only standard rehabilitation, and one was transferred to another facility. Compliance with active therapy was high in both groups. Excluding the 3 patients who did not receive the intervention or withdrew before the end of therapy, the mean total time in active therapy was 1769 minutes for NAT $(98 \%$ of target) and 1801 for COT (100\% of target). Two patients in the NAT group required minimal assistance from the therapist to initiate movement in the first few sessions but were subsequently able to complete the training on their own. Twenty-one patients 
completed the post-training day 3 assessment (88\%), 20 completed the post-training day 90 assessment (83\%), and 19 completed the day 180 assessment $(79 \%)$.

\section{Clinical outcomes}

Our primary outcome was the change in FM-UE score from baseline (pretraining) to post-training day 3 . There was no significant difference between NAT and COT groups in our primary outcome of FM-UE changes from baseline to day 3 posttraining (difference 1.34, standard error [SE] 5.15, $p=0.797$ ), or in FM-UE changes from baseline to day 90 (difference -3.28 , SE 5.26, $p=0.316$ ) and day 180 (difference -0.09 , SE 5.38, $p=0.132$ ) post-training (Table 2 ). There was similarly no significant difference between NAT and COT for the change in ARAT from baseline to day 3, day 90, or day 180 post-training (Table 2). Grip strength and SIS hand also showed no between-group differences in change from baseline at any timepoint (Table 2).

\section{Reaching kinematics}

We calculated the average squared Mahalanobis distance $\left(A M D^{2}\right)$ for reaching trajectories performed with the paretic arm at the four timepoints, compared to a reference population of neurologically-healthy control subjects. There were 2 patients in the COT group whose reaching kinematic data were excluded from analysis because there were too few movements in the baseline session that were suitable for inclusion in the analysis. An additional 2 patients were missing baseline assessments of reaching kinematics ( 1 in each group) and therefore were not included in the analysis of change scores from baseline. Of the remaining patients, day 90 data were missing for 2 
subjects and day 180 data were missing for 2 patients, due to time constraints or withdrawal from the study. There was improvement in reach kinematics across the intervention period but there was no significant difference in the change in $A M D^{2}$ from baseline between NAT and COT groups at day 3 , day 90 , or day 180 post-training (Table 2). There was a significant correlation between the $\mathrm{AMD}^{2}$ and the ARAT ( $p=$ $0.028)$ but not the FM-UE $(p=0.622)$.

Finger strength and individuation

Maximum voluntary force and finger individuation index were calculated for the more affected arm at the four timepoints as described above. 1 patient in the NAT group did not complete the tasks at baseline and was excluded from analysis. There were also data missing for 4 patients at post-training day 90 ( 2 in NAT and 2 in COT), and 5 patients at post-training day 180 ( 2 in NAT and 3 in COT). We found no significant between-group difference in the change in MVC or individuation index from baseline to any post-training timepoint. (Table 2 ).

Comparison of intensive therapy to the historical cohort (HC)

In an exploratory analysis, we compared the change in FM-UE and ARAT from baseline to post-training day 3 in our SMARTS2 study cohort (NAT and COT groups combined) to changes across a similar timeframe with usual care in a historical cohort from the EXPLICIT-stroke study ${ }^{25}$. Patients in SMARTS2 were matched by time poststroke and severity (FM-UE and ARAT) with patients from EXPLICIT. We observed a significant benefit in upper limb activity (ARAT difference 7.33, SE 2.88 pts, $p=0.011$ ) 


\begin{abstract}
but not for impairment (FM-UE difference 1.44, SE 2.57, $p=0.564$ ) with the intensive therapy provided in SMARTS2 compared with usual care (Figure 3).
\end{abstract}

\title{
Adverse events
}

There were a total of 55 adverse events (AEs) that occurred in 13 patients during the study. There were 4 serious adverse events in the COT gorup that were unrelated to the study procedures, (2 falls resulting hospitalization and 2 unrelated medical conditions). Of the 51 non-serious AEs, 23 (42\%) occurred in the NAT group and 32 $(58 \%)$ occurred in the COT group. In the NAT group, 5 AEs were probably related (neck pain, fatigue in 3 patients, and bruising) and 6 AEs were possibly related (eye pain in 2 patients, headache, nausea, worsened ataxia, and a fall) to study procedures. In the COT group, two AEs were definitely related (wrist pain in 2 sessions), 1 probably related (pain), and two possibly related (pain, fall) to study procedures.

\section{DISCUSSION}

In this randomized, single-blinded proof-of-concept trial we sought to test the idea, inspired by studies in non-human primates, that high-intensity and high-dose upper limb therapy focused on movement quality rather than task accomplishment, and delivered early after stroke, would reduce motor impairment more than usual care does. We tested this main idea by taking two distinct approaches. The first was administering high doses of conventional upper limb therapy. The second was a new immersive animated experience that centers on a proprietary form of animation designed to promote playful exploration of high quality continuous 3D arm movements ${ }^{13}$. Here we 
found that both approaches led to similar changes in the FM-UE, ARAT, reaching kinematics, finger strength, and finger individuation. Looking at a historical cohort, we found that both approaches were superior to usual care with respect to the ARAT but not the FM-UE. Unfortunately, hand and planar kinematic measures were not available for the historical cohort.

Conventional occupational therapy (COT) mainly emphasizes repetitive taskoriented training (TOT $)^{26,27}$, an approach predicated on practice schedules based on motor learning principles. Not much time is dedicated to the upper limb in regular therapy sessions ${ }^{12}$, which means that TOT focuses more on compensatory movements for task accomplishment. That said, there is nothing inherent to COT that precludes a switch in emphasis to movement quality, especially if therapists are given more time with the patient, as they were in SMARTS2. In three recent studies in patients with chronic stroke, large gains in both the ARAT (or other activity-level measure) and the FM-UE were seen when patients were provided with either five or six hours of upper limb therapy a day for five days/week for three, six or twelve weeks ${ }^{3-5}$. Clearly these are very high intensities and doses of therapy. In two of the studies ${ }^{4,5}$, the authors explicitly state that they wanted to make "movement practice as close to normal as possible", and did so by progressing from single-joint to two-joint movements, then assembling these into task components and finally practicing performance of the full task. It is evident that they combined the more traditional neurophysiological approach, which focuses on movement quality, with TOT. The therapists in our study took a comparable approach in the COT group, as outlined in the methods section. In fact, they explicitly stated on 
questioning that they were able to focus on movement quality precisely because they had more time with the patient.

The NAT and COT groups showed comparable changes in ARAT scores. These changes were significantly greater than the changes seen in the historical control group receiving usual care over the same time period. The ARAT is a valid and responsive measure of upper limb activity on the ICF scale ${ }^{28,29}$ and its components have considerable overlap with the reach and grasp tasks in non-human primate experiments investigating motor recovery. Indeed, the ARAT correlates well with kinematic measures of reach and grasp ${ }^{30}$.

We argue here that the changes in the ARAT in the NAT and COT groups are an indication of true improvement in the quality of arm and hand motor control and not just compensation, even though performance on items scored less than 3 (i.e., "normal") can include compensatory movements. First, we know that the ARAT can show, just like the FM-UE, changes, as we saw here (13.4 and 14.70 points from baseline to day 3 post-training in NAT and COT groups, respectively), that are larger earlier compared to later after stroke ${ }^{31}$. That ARAT changes are greater when high-dose therapy is given earlier than later means suggests that they, at least in part, reflect true restitution and not just learned compensation. Second, if the two intervention groups were just being trained to compensate better than usual care, then this must be because they learned to compensate during the intervention. This would not be possible for the NAT group, however, because there was no-prehension functional reach and grasp training-of any kind - the virtual dolphins were steered with the arm only.. Third, large improvements in activity measures have been seen with intense and high dose COT in patients with 
chronic stroke $e^{5,6}$. These changes seem to be dose-dependent, only becoming large when 90 hours or more of treatment are given ${ }^{3-5}$. In contrast, 32 hours does not lead to large ARAT changes ${ }^{31}$. If large ARAT changes were just due to optimizing compensatory strategies it seems unlikely that they would not also be seen after 32 hours of COT. Finally, here we found a significant correlation, as have others ${ }^{30,32}$, between improvement in the quality of arm kinematics and the ARAT. Thus, like in nonhuman primates ${ }^{8}$, we conclude that intense and high-dose upper limb therapy focused on movement quality can at least partially restore motor control in the upper limb in the subacute period after stroke; it does not just train compensatory movements.

Notably, we did not see an increase in the FM-UE, our primary outcome measure, beyond what was seen in the usual care historical control group. Of course, as we were studying subacute rather than chronic stroke, there were large changes in the FM-UE due to spontaneous recovery but we were not able to augment them with either of our interventions. It is always possible that this is a false negative result given the low $n$, but a previous study of early intense and high-dose upper limb therapy, in this case constraint-induced movement therapy, also reported a dissociation between the ARAT and the FM-UE ${ }^{25}$.

It is possible for ARAT improvements to reflect true changes in motor control and yet not be detected by the FM-UE score due to differences in emphasis for the two scales. For example, a patient who has regained active range of movement in the shoulder/elbow but has persistent difficulty with out of synergy movements (e.g., unable to initiate shoulder flexion or abduction without bending the elbow, which would confer a score of "0" on these FM-UE items) may nevertheless improve on the ARAT by gaining 
the ability to reach the top shelf through improved shoulder flexion and elbow extension. Another situation in which one may see a dissociation between FM-UE and ARAT changes is a patient who primarily regains distal dexterity, which is weighted more heavily on the ARAT than the FM-UE.

The differential responsiveness of the FM-UE and the ARAT to an intervention is not altogether surprising. FM-UE and ARAT measure different constructs of the ICF model, reflecting the levels of body function and activity, respectively. In addition, the FM-UE scale was primarily devised to quantify post-stroke synergies over the course of motor recovery ${ }^{33}$ whereas the ARAT emphasizes assessment of prehension (combined reach and grasp) during more functional tasks ${ }^{29,34}$. In other words, one primarily targets a positive sign and the other a negative sign of the upper motor neuron syndrome ${ }^{8}$. That being said, synergies will intrude on a functional task, especially in the absence of arm weight support ${ }^{35,36}$, which is why the two measures often correlate with each other ${ }^{37,38}$. It is unfortunate that the FM-UE has come to be considered synonymous with overall impairment after stroke, even though it was designed to assess mostly a single component of impairment, namely synergies, over strength or dexterity. This is problematic because thus far it seems that the positive and negative symptoms of stroke respond differently to interventions in the sub-acute period. In this study, we saw significant improvement in ARAT but not in FM-UE with our intensive movement qualityfocused training, and a similar finding was reported when extra sessions of constraintinduced movement therapy were added in the sub-acute period in the EXPLICIT trial ${ }^{25}$. The divergence of ARAT and FM-UE in comparison to usual care was descriptive rather than statistical, due to the challenges in performing a robust statistical comparison 
between values of different scales, the dependence between tests of each outcome, and the repeated matching framework that was used to compare recovery between groups. Nevertheless, the differences in $p$-values supports a divergence for FM-UE $(p=$ $0.564)$ and ARAT $(p=0.011)$. It is to be hoped that new kinematic measures will soon be developed that can distinguish arm dexterity/quality of motor control from both synergies and compensation during performance of 3D functional tasks ${ }^{39,40}$. This is of utmost importance because hemiparesis in humans appears to be both a deficit disorder related to damage to the corticospinal tract ${ }^{41,42}$ and a movement disorder, perhaps related to upregulation of the reticulospinal tract ${ }^{43-45}$. These positive and negative signs of hemiparesis will likely need distinct forms of intervention.

Our findings for the NAT and COT groups are congruent with what has been reported in many recent neurorehabilitation studies and trials - both the novel intervention group and the control group show similar, and often large, treatment responses. This has been taken as evidence that new technological or pharmacological interventions do not outperform higher intensities and doses of conventional therapy or, by extension, usual clinical care. In a recent review of 15 neurorehabilitation trials conducted in the last five years it is stated: "There is no clear evidence that interventions tested in large multicenter stroke rehabilitation trials are superior to current care. Furthermore, patients benefited from both the experimental and control interventions at both the subacute and chronic stages" 46 . The crucial point being missed here, however, is that control interventions in clinical trials often consist of more, and sometimes considerably more, conventional therapy than is usually given during regular clinical care. This is certainly the case in our study where patients in the COT group 
received two hours a day of therapy for five consecutive days over three weeks. Because of the greatly increased time available, both NAT and COT were able to increase the emphasis on movement quality and discourage use of compensatory movements. At the very least, one can safely say that there was more movementquality-based training than in the historical cohort. In addition, trials tend to select for patients with less comorbidity and fewer cognitive deficits, which allows them to receive higher doses of usual care. Therefore, the positive results for controls in trials do not imply that usual conventional care is equally efficacious to the novel intervention. Indeed, trials that have directly compared higher doses with usual doses of conventional care have found a difference between them ${ }^{47-49}$. Thus, the fact that a new approach, like the NAT here, is not significantly different from high doses of COT should be taken as a reason for optimism. This implies that the new intervention must possess an active ingredient that potentially could be further optimized in terms of efficacy, efficiency, costeffectiveness, and scalability. This situation can be considered analogous to what happened in the evolution of thrombectomy for acute stroke. In 2013, three trials showed no benefit of thrombectomy over usual care ${ }^{50-52}$. By 2015 , five trials showed superiority for thrombectomy ${ }^{53}$. What happened? The two main reasons were choice of the correct technology for clot removal and a change in protocol design.

As with post-stroke thrombolysis, it is possible that we are on the cusp of a change in the delivery and efficacy of upper limb neurorehabilitation. In this case, the correct choice would be to move toward more immersive experiences to promote intense exploratory training with a focus on movement quality. The protocol change would be, as in SMARTS2, to encourage exploratory multi-joint movements outside of a 
task context for at least two hours a day. Thus, based on the results here, we suggest that an alternative to just increasing the amount of time available to administer COT, is to devise technology-based solutions are scalable and that make it easier and more enjoyable to deliver higher doses and intensities of impairment-focused therapy ${ }^{54}$. It should also be emphasized, that even though in this small study we found no significant difference between COT and NAT for delivery of higher doses of intense upper limb therapy, it does not need to be either/or. It may turn out that the two approaches can complement each other. COT could be considered analogous to drills in sport, for example practicing backhands in tennis for an hour. NAT could be the holistic approach where you combine all the components into a full game.

Compliance with therapy was high in both NAT and COT groups, reaching $98 \%$ and $100 \%$ of targeted time on task, respectively. Overall both interventions were safe, with no serious adverse events related to study procedures. There were, however, more adverse events in the COT group than the NAT group. Fatigue was reported more often in the NAT group, which therapists did not always perceive to be a negative because the therapy was designed to be challenging. Other side effects in the NAT group such as transient headache and pain have been reported previously with game-based interventions and are not unexpected with high intensity training ${ }^{55}$.

This study clearly has a number of limitations. First, the number of patients in this proof-of-concept trial was low. Indeed, we recruited only half the number of patients we anticipated. This is attributable both to our inclusion/exclusion criteria and the challenges of providing two hours of time-on-task upper limb therapy, five days a week beginning in the first six weeks after stroke in addition to usual care ${ }^{56,57}$. Furthermore, 
incorporation of mechanical thrombectomy as standard of care led to fewer patients with severe to moderate motor deficits. Second, we were only able to begin our interventions after patients were discharged from in-patient acute rehabilitation at the sites in the United States due to the challenges of delivering high doses of therapy in addition to standard care in the inpatient rehabilitation setting as well as the need for short length of stay ${ }^{58}$; the average start time was therefore about three weeks post-stroke. From our previous work, we have shown that the time window of heightened neuroplasticity responsible for spontaneous recovery, and perhaps for enhanced training-related improvement that takes advantage of this heightened neuroplasticity, might be as short as 5 weeks ${ }^{15}$. This difficulty with our enrollment time window attests to the continuing challenge of conducting neurorehabilitation trials in this early time period after stroke ${ }^{46,58,59}$. Third, we did not track amount of upper limb activity at home during this study, thus are unable to quantify whether either of the training interventions influenced amount of limb use in the real world. Fourth, we had to use a historical usual care group, albeit an extensive and well-matched one ${ }^{25}$. This was necessary because we were not able to ask patients to enroll in a trial offering three weeks of extra care with the chance that they would end up in the control group that got no extra therapy but would nevertheless require them to make trips to the hospital for assessment. Given our strict time window offering the active intervention later was not an option. Another point, as we made above, is that in clinical trials, the control intervention is most often not "usual" care but an amplified and often unrealistic version of it ${ }^{46}$. Here we were fortunate that a cohort existed that delivered care of the upper limb that was close to what patients actually receive in the subacute post-stroke period. 


\section{CONCLUSIONS}

Increasing the dose and intensity of upper limb rehabilitation training early after stroke, with focus on movement quality, led to gains beyond those seen with usual care. This additional improvement was achieved either by having therapists provide much more COT or with a novel exploratory animation-based approach with exoskeletal weight support. This is exciting, as it suggests that an immersive animation-based experience combined with weight-support might pave the way forward for providing high doses of upper limb rehabilitation focused on movement quality in a more efficient, enjoyable, and scalable way at any time post-stroke.

\section{ACKNOWLEDGMENTS}

This study was principally funded by the James S. McDonnell Foundation Grant 220020220 (J.W.K.). Additional funding came from the P\&K Pühringer Foundation (A.R.L.), Sheikh Khalifa Stroke Institute (SKSI) (J.W.K., O.A., P.R., R.S.) and the Neurology and PMR Departments at the Johns Hopkins University School of Medicine. J.G.'s work was supported in part by NIH grant R01NS097423. The EXPLICIT-stroke consortium and the EXPLICIT-stroke cohort were funded by The Netherlands Organization for Health Research and Development (ZonMw Grant No. 89000001). JWK, OA, and PR are co-inventors of the underlying dolphin technology, which has now been licensed to MSquare Healthcare (a MindMaze Inc. Company) and is available as an approved device called MindPod Dolphin. 


\section{REFERENCES}

1. Prabhakaran S, Zarahn E, Riley C, et al. Inter-individual variability in the capacity for motor recovery after ischemic stroke. Neurorehabil Neural Repair. 2008;22(1):64-71.

2. Byblow WD, Stinear CM, Barber PA, Petoe MA, Ackerley SJ. Proportional recovery after stroke depends on corticomotor integrity. Ann Neurol. 2015;78(6):848-859.

3. Ward NS, Brander F, Kelly K. Intensive upper limb neurorehabilitation in chronic stroke: outcomes from the Queen Square programme. J Neurol Neurosurg Psychiatry. 2019;90(5):498-506.

4. Daly JJ, McCabe JP, Holcomb J, Monkiewicz M, Gansen J, Pundik S. Long-Dose Intensive Therapy Is Necessary for Strong, Clinically Significant, Upper Limb Functional Gains and Retained Gains in Severe/Moderate Chronic Stroke. Neurorehabil Neural Repair. 2019;33(7):523-537.

5. McCabe J, Monkiewicz M, Holcomb J, Pundik S, Daly JJ. Comparison of robotics, functional electrical stimulation, and motor learning methods for treatment of persistent upper extremity dysfunction after stroke: a randomized controlled trial. Arch Phys Med Rehabil. 2015;96(6):981-990.

6. Mawase F, Cherry-Allen KM, Xu J, Anaya M, Uehara S, Celnik P. Pushing the rehabiltiation boundaries: hand motor impairment can be reduced in chronic stroke Neurorehabil Neural Repair. 2020; In press.

7. Darling WG, Pizzimenti MA, Morecraft RJ. Functional recovery following motor cortex lesions in non-human primates: experimental implications for human stroke patients. $J$ Integr Neurosci. 2011;10(3):353-384.

8. Krakauer JW, Carmichael ST. Broken Movement: The Neurobiology of Motor Recovery After STroke. Cambridge, MA: The MIT Press; 2017.

9. Nudo RJ, Wise BM, SiFuentes F, Milliken GW. Neural substrates for the effects of rehabilitative training on motor recovery after ischemic infarct. Science. 1996;272:17911794.

10. Murata $\mathrm{Y}$, Higo N, Oishi T, et al. Effects of motor training on the recovery of manual dexterity after primary motor cortex lesion in macaque monkeys. $J$ Neurophysiol. 2008;99(2):773-786.

11. Dobkin $\mathrm{BH}$. Clinical practice. Rehabilitation after stroke. N Engl J Med. 2005;352(16):1677-1684.

12. Lang CE, Macdonald JR, Reisman DS, et al. Observation of amounts of movement practice provided during stroke rehabilitation. Arch Phys Med Rehabil. 2009;90(10):1692-1698.

13. Krakauer JW, Cortes JC. A non-task-oriented approach based on high-dose playful movement exploration for rehabilitation of the upper limb early after stroke: A proposal. NeuroRehabilitation. 2018;43(1):31-40.

14. Biernaskie J, Chernenko G, Corbett D. Efficacy of rehabilitative experience declines with time after focal ischemic brain injury. J Neurosci. 2004;24(5):1245-1254.

15. Cortes JC, Goldsmith J, Harran MD, et al. A Short and Distinct Time Window for Recovery of Arm Motor Control Early After Stroke Revealed With a Global Measure of Trajectory Kinematics. Neurorehabil Neural Repair. 2017;31(6):552-560.

16. Gowland C, Stratford P, Ward M, et al. Measuring physical impairment and disability with the Chedoke-McMaster Stroke Assessment. Stroke. 1993;24(1):58-63.

17. Nasreddine ZS, Phillips NA, Bedirian V, et al. The Montreal Cognitive Assessment, MoCA: a brief screening tool for mild cognitive impairment. J Am Geriatr Soc. 2005;53(4):695-699. 
18. Rothi LJG, Raymer AM, Heilman KM. Apraxia: The neuropsychology of action. . Hove, U.K.: Psychology Press; 1997.

19. Halligan $P$, Wilson $B$, Cockburn J. A short screening test for visual neglect in stroke patients. Int Disabil Stud. 1990;12(3):95-99.

20. Beck AT, Steer RA, Brown GK. Manual for the Beck Depression Inventory-II. San Antonio, TX: Psychological Corporation 1996.

21. Gladstone DJ, Danells CJ, Black SE. The fugl-meyer assessment of motor recovery after stroke: a critical review of its measurement properties. Neurorehabil Neural Repair. 2002;16(3):232-240.

22. Lang CE, Edwards DF, Birkenmeier RL, Dromerick AW. Estimating minimal clinically important differences of upper-extremity measures early after stroke. Arch Phys Med Rehabil. 2008;89(9):1693-1700.

23. Lai SM, Studenski S, Duncan PW, Perera S. Persisting consequences of stroke measured by the Stroke Impact Scale. Stroke. 2002;33(7):1840-1844.

24. Xu J, Ejaz N, Hertler B, et al. Separable systems for recovery of finger strength and control after stroke. J Neurophysiol. 2017;118(2):1151-1163.

25. Kwakkel G, Winters C, van Wegen EE, et al. Effects of Unilateral Upper Limb Training in Two Distinct Prognostic Groups Early After Stroke: The EXPLICIT-Stroke Randomized Clinical Trial. Neurorehabil Neural Repair. 2016;30(9):804-816.

26. Winstein CJ, Wolf SL, Dromerick AW, et al. Effect of a Task-Oriented Rehabilitation Program on Upper Extremity Recovery Following Motor Stroke: The ICARE Randomized Clinical Trial. Jama. 2016;315(6):571-581.

27. Winstein CJ, Stein J, Arena R, et al. Guidelines for Adult Stroke Rehabilitation and Recovery: A Guideline for Healthcare Professionals From the American Heart Association/American Stroke Association. Stroke. 2016;47(6):e98-e169.

28. van der Lee JH, Beckerman H, Lankhorst GJ, Bouter LM. The responsiveness of the action research arm test and the Fugl-Meyer Assessment scale in chronic stroke patients. Journal of Rehabilitation Medicine. 2001;33(3):110-113.

29. Yozbatiran N, Der-Yeghiaian L, Cramer SC. A standardized approach to performing the action research arm test. Neurorehabil Neural Repair. 2008;22(1):78-90.

30. Lang CE, Wagner JM, Dromerick AW, Edwards DF. Measurement of upper-extremity function early after stroke: properties of the action research arm test. Arch Phys Med Rehabil. 2006;87(12):1605-1610.

31. Lang CE, Strube MJ, Bland MD, et al. Dose response of task-specific upper limb training in people at least 6 months poststroke: A phase II, single-blind, randomized, controlled trial. Ann Neurol. 2016;80(3):342-354.

32. Alt Murphy M, Willen C, Sunnerhagen KS. Responsiveness of upper extremity kinematic measures and clinical improvement during the first three months after stroke.

Neurorehabil Neural Repair. 2013;27(9):844-853.

33. Fugl-Meyer AR, Jaasko L, Leyman I, Olsson S, Steglind S. The post-stroke hemiplegic patient. 1. a method for evaluation of physical performance. Scand J Rehabil Med. 1975;7(1):13-31.

34. Lyle RC. A performance test for assessment of upper limb function in physical rehabilitation treatment and research. Int J Rehabil Res. 1981;4(4):483-492.

35. Zackowski KM, Dromerick AW, Sahrmann SA, Thach WT, Bastian AJ. How do strength, sensation, spasticity and joint individuation relate to the reaching deficits of people with chronic hemiparesis? Brain. 2004;127(Pt 5):1035-1046.

36. Lan Y, Yao J, Dewald JPA. The Impact of Shoulder Abduction Loading on Volitional Hand Opening and Grasping in Chronic Hemiparetic Stroke. Neurorehabil Neural Repair. 2017;31(6):521-529. 
37. Platz T, Pinkowski C, van Wijck F, Kim IH, di Bella P, Johnson G. Reliability and validity of arm function assessment with standardized guidelines for the Fugl-Meyer Test, Action Research Arm Test and Box and Block Test: a multicentre study. Clin Rehabil. 2005;19(4):404-411.

38. Wei XJ, Tong KY, Hu XL. The responsiveness and correlation between Fugl-Meyer Assessment, Motor Status Scale, and the Action Research Arm Test in chronic stroke with upper-extremity rehabilitation robotic training. Int J Rehabil Res. 2011;34(4):349356.

39. Kwakkel G, Van Wegen E, Burridge JH, et al. Standardized measurement of quality of upper limb movement after stroke: Consensus-based core recommendations from the Second Stroke Recovery and Rehabilitation Roundtable. Int J Stroke. 2019;14(8):783791.

40. Schwarz A, Kanzler CM, Lambercy O, Luft AR, Veerbeek JM. Systematic Review on Kinematic Assessments of Upper Limb Movements After Stroke. Stroke. 2019;50(3):718-727.

41. Tower SS. Pyramidal lesion in the monkey. Brain. 1940;63(1):36-90.

42. Beebe JA, Lang CE. Absence of a proximal to distal gradient of motor deficits in the upper extremity early after stroke. Clin Neurophysiol. 2008;119(9):2074-2085.

43. Owen M, Ingo C, Dewald JPA. Upper Extremity Motor Impairments and Microstructural Changes in Bulbospinal Pathways in Chronic Hemiparetic Stroke. Front Neurol. 2017;8:257.

44. Ellis MD, Drogos J, Carmona C, Keller T, Dewald JP. Neck rotation modulates flexion synergy torques, indicating an ipsilateral reticulospinal source for impairment in stroke. $J$ Neurophysiol. 2012;108(11):3096-3104.

45. Zaaimi B, Edgley SA, Soteropoulos DS, Baker SN. Changes in descending motor pathway connectivity after corticospinal tract lesion in macaque monkey. Brain. 2012;135(Pt 7):2277-2289.

46. Stinear CM, Lang CE, Zeiler S, Byblow WD. Advances and challenges in stroke rehabilitation. Lancet Neurol. 2020;19(4):348-360.

47. Lo AC, Guarino PD, Richards LG, et al. Robot-assisted therapy for long-term upper-limb impairment after stroke. N Engl J Med. 2010;362(19):1772-1783.

48. Rodgers $\mathrm{H}$, Bosomworth $\mathrm{H}$, Krebs $\mathrm{HI}$, et al. Robot assisted training for the upper limb after stroke (RATULS): a multicentre randomised controlled trial. Lancet. 2019;394(10192):51-62.

49. Wolf SL, Winstein CJ, Miller JP, et al. Effect of constraint-induced movement therapy on upper extremity function 3 to 9 months after stroke: the EXCITE randomized clinical trial. Jama. 2006;296(17):2095-2104.

50. Kidwell CS, Jahan R, Gornbein J, et al. A trial of imaging selection and endovascular treatment for ischemic stroke. N Engl J Med. 2013;368(10):914-923.

51. Broderick JP, Palesch YY, Demchuk AM, et al. Endovascular therapy after intravenous tPA versus t-PA alone for stroke. N Engl J Med. 2013;368(10):893-903.

52. Ciccone A, Valvassori $L$, Nichelatti $M$, et al. Endovascular treatment for acute ischemic stroke. N Engl J Med. 2013;368(10):904-913.

53. Moore JM, Griessenauer CJ, Gupta R, et al. Landmark papers in cerebrovascular neurosurgery 2015. Clin Neurol Neurosurg. 2016;148:22-28.

54. Wuennemann MJ, Mackenzie SW, Lane HP, et al. Dose and staffing comparison study of upper limb device-assisted therapy. NeuroRehabilitation. 2020.

55. Laver KE, Lange B, George S, Deutsch JE, Saposnik G, Crotty M. Virtual reality for stroke rehabilitation. Cochrane Database Syst Rev. 2017;11:CD008349. 
56. Cramer SC, Wolf SL, Adams HP, Jr., et al. Stroke Recovery and Rehabilitation Research: Issues, Opportunities, and the National Institutes of Health StrokeNet. Stroke. 2017;48(3):813-819.

57. Stinear CM. Stroke rehabilitation research needs to be different to make a difference. F1000Res. 2016;5.

58. Raghavan P. Research in the Acute Rehabilitation Setting: a Bridge Too Far? Curr Neurol Neurosci Rep. 2019;19(1):4.

59. Stinear C, Ackerley S, Byblow W. Rehabilitation is initiated early after stroke, but most motor rehabilitation trials are not: a systematic review. Stroke; a journal of cerebral circulation. 2013;44(7):2039-2045. 


\section{Manuscript: NNR-20-0383 \\ Response to reviewers}

We thank the reviewers for their comments and have addressed them point-bypoint below and in the revised manuscript.

1. The authors appear to be agreeing with my point that the divergence in ARAT and FM is descriptive. However, they are still keen to state 'Nevertheless, our results support a divergence for FM-UE $(p=0.564)$ and ARAT $(p=0.011)$.' based on the fact that the two $p$-values are not 'qualitatively similar'. I'm afraid this is not justified and this sentence should not remain. I agree that the authors can keep their discussion of FM and ARAT differences because it's interesting, but I want to remind them that this was not part of the study aims.

Response: We agree and have removed the sentence 'Nevertheless, our results support a divergence for FM-UE $(p=0.564)$ and ARAT $(p=$ 0.011).'

2. The removal of prehension addresses the last point

Response: We have removed the word "prehension" (p.19) and have changed it to "functional reach and grasp training". In the description of the ARAT (p.21) we have also clarified that "ARAT emphasizes assessment of prehension (combined reach and grasp) during more functional tasks." 
Table 1. Participant characteristics at baseline

\begin{tabular}{|c|c|c|c|}
\hline & $\begin{array}{c}\text { NAT } \\
(n=13)\end{array}$ & $\begin{array}{c}\text { COT } \\
(n=11)\end{array}$ & $p$-value \\
\hline Age in years (SD) & $62.0(10.4)$ & $64.4(14.0)$ & 0.640 \\
\hline Gender male (\%) & $5(38.5 \%)$ & $6(54.5 \%)$ & 0.706 \\
\hline Nondominant affected & $7(53.8 \%)$ & $5(45.5 \%)$ & 1.000 \\
\hline Received tPA (\%) & $10(76.9 \%)$ & $7(63.6 \%)$ & 0.793 \\
\hline Thrombectomy (\%) & $2(15.4 \%)$ & $3(27.3 \%)$ & 0.834 \\
\hline $\begin{array}{l}\text { Days between stroke } \\
\text { onset and baseline } \\
\text { assessment } \\
\text { (median [IQR]) }\end{array}$ & $12.0,33.0]$ & $14.0[12.5,35.5]$ & 0.663 \\
\hline NIHSS (median [IQR]) & $6.0[6.0,10.0]$ & $6.0[5.5,9.5]$ & 0.859 \\
\hline $\begin{array}{l}\text { Florida Apraxia Battery } \\
\text { (median [IQR]) }\end{array}$ & $15[15,15]$ & $14[12.5,15]$ & 0.031 \\
\hline Hemineglect (\%) & $1(7.7 \%)$ & $1(10.0 \%)$ & 1.000 \\
\hline $\begin{array}{l}\text { Abnormal } \\
\text { proprioception (\%) }\end{array}$ & $2(15.4 \%)$ & $5(45.5 \%)$ & 0.244 \\
\hline $\begin{array}{l}\text { Beck Depression } \\
\text { (median [IQR]) }\end{array}$ & $17[11,19]$ & $5[3,8]$ & 0.025 \\
\hline Taking SSRI (\%) & $10(76.9 \%)$ & $4(36.4 \%)$ & 0.111 \\
\hline $\begin{array}{l}\text { Baseline FM-UE } \\
\text { (median [IQR]) }\end{array}$ & $23.8(12.1)$ & $22.2(8.7)$ & 0.562 \\
\hline $\begin{array}{l}\text { Baseline ARAT } \\
\text { (median [IQR]) }\end{array}$ & $10.0[3.0,33.0]$ & $9.0[3.0,21.5]$ & 0.640 \\
\hline
\end{tabular}

NAT = neuroanimation therapy. COT = conventional occupational therapy. tPA = tissue plasminogen activator. NIHSS $=$ National Institutes of Health Stroke Scale. Florida Apraxia Battery scores range 0-15 with a lower score indicating worse apraxia. SSRI=selective serotonin reuptake inhibitor. FM-UE=Fugl-Meyer Upper Extremity motor score. ARAT=Action Research Arm Test 


\section{Baseline to day 3}

\begin{tabular}{lllll} 
& NAT & COT & \multicolumn{2}{l}{ Difference (SE) } \\
& 12.5 & 13.4 & $0.9(3.7)$ & 0.797 \\
FM-UE & 13.4 & 14.7 & $1.3(5.1)$ & 0.795 \\
ARAT & 8.57 & 2.63 & $-5.93(6.27)$ & 0.344 \\
$\begin{array}{l}\text { Grip strength } \\
\text { (lbs) }\end{array}$ & & & & \\
SIS hand & 21.8 & 14.0 & $-7.8(11.1)$ & 0.480 \\
$\begin{array}{l}\text { Reaching } \\
\text { AMD2 }\end{array}$ & -30.87 & -26.67 & $4.20(14.11)$ & 0.766 \\
$\begin{array}{l}\text { Finger MVF } \\
\text { (N) }\end{array}$ & 0.14 & 0.20 & $0.06(0.13)$ & 0.647 \\
$\begin{array}{l}\text { Finger } \\
\text { Individuation }\end{array}$ & 0.22 & 0.19 & $-0.03(0.12)$ & 0.780
\end{tabular}

\section{Baseline to day 90}

$\begin{array}{llll}\text { NAT } & \text { COT } & \text { Difference } & \mathbf{p} \\ 18.2 & 14.4 & -3.8(3.8) & 0.316 \\ 19.8 & 16.5 & -3.3(5.3) & 0.532 \\ 12.39 & 8.23 & -4.16(6.38) & 0.515 \\ & & & \\ 36.8 & 20.1 & -16.7(11.3) & 0.139 \\ -39.73 & -50.12 & -10.39(14.75) & 0.482 \\ 0.26 & 0.33 & 0.07(0.14) & 0.632 \\ 0.31 & 0.31 & 0.00(0.13) & 0.999\end{array}$

\section{Baseline to day 180}

$\begin{array}{llll}\text { NAT } & \text { COT } & \text { Difference } & \mathbf{p} \\ 19.6 & 13.8 & -5.8(3.8) & 0.132 \\ 21.7 & 21.6 & -0.1(5.4) & 0.986 \\ 16.36 & 7.26 & -9.10(6.51) & 0.162 \\ & & & \\ 42.3 & 29.9 & -12.4(11.5) & 0.282 \\ -41.42 & -48.84 & -7.42(15.03) & 0.622 \\ & & & \\ 0.24 & 0.24 & 0.00(0.14) & 0.992 \\ 0.36 & 0.31 & -0.05(0.14) & 0.717\end{array}$

Table 2. Results of mixed model estimates of changes from baseline to post-training days 3, 90, and 180 .

There were no significant differences between NAT and OT groups in any of the primary or secondary outcome measures. Abbreviations: NAT=neuroanimation therapy; COT=conventional occupational therapy; $S E=$ standard error; $p=p$-value; FMUE=Fugl-Meyer Upper Extremity motor assessment; ARAT=Action Research Arm Test; SIS hand=Stroke Impact Scale v.2 hand domain; $\mathrm{AMD}^{2}=$ average squared Mahalanobis distance; $\mathrm{MVF}=$ maximum voluntary force. 


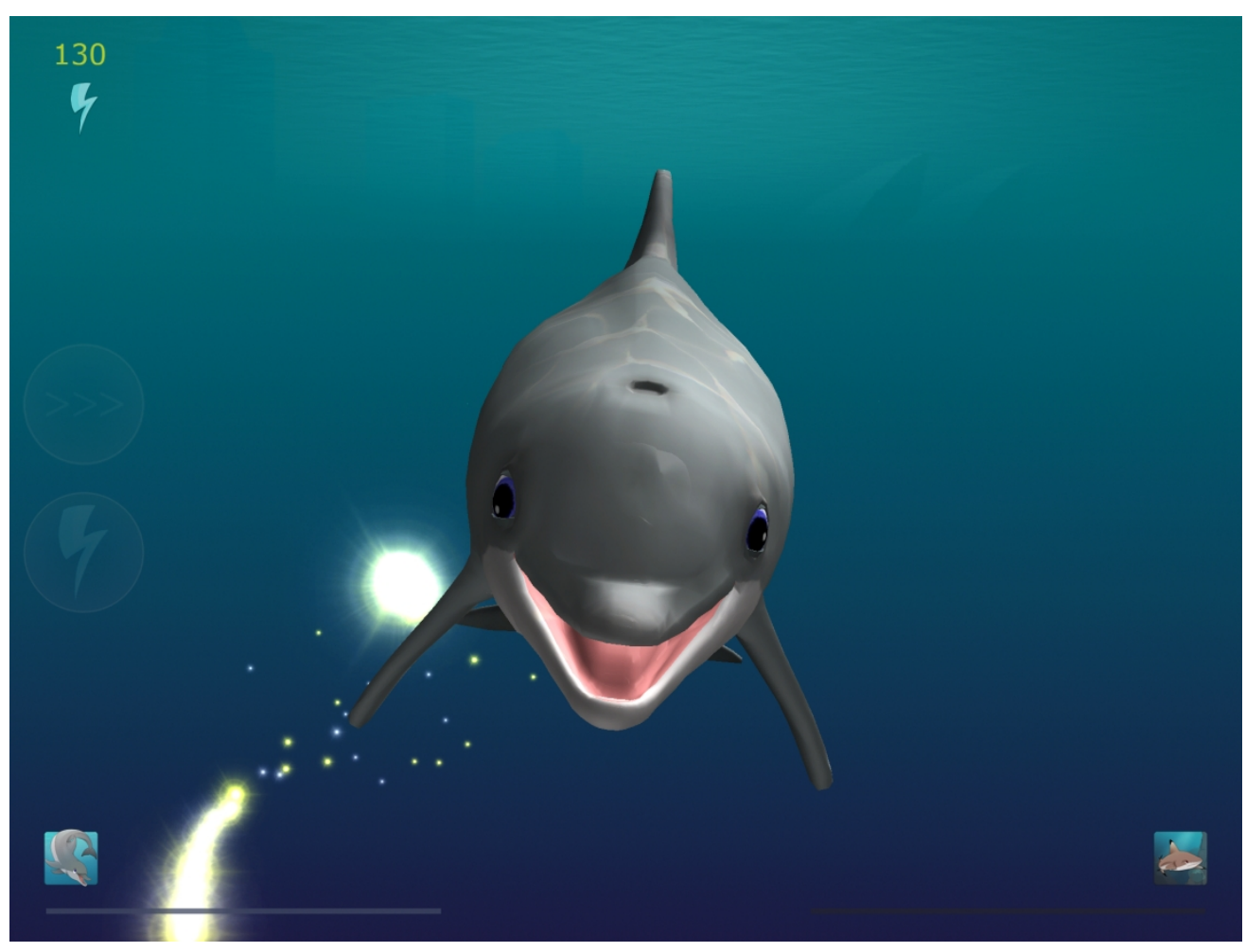

Figure 1. Participants in the neuroanimation therapy group played the MindPod Dolphin game while their arm was unweighted by the Armeo $®$ Power exoskeleton device.

$722 \times 541 \mathrm{~mm}(72 \times 72 \mathrm{DPI})$ 


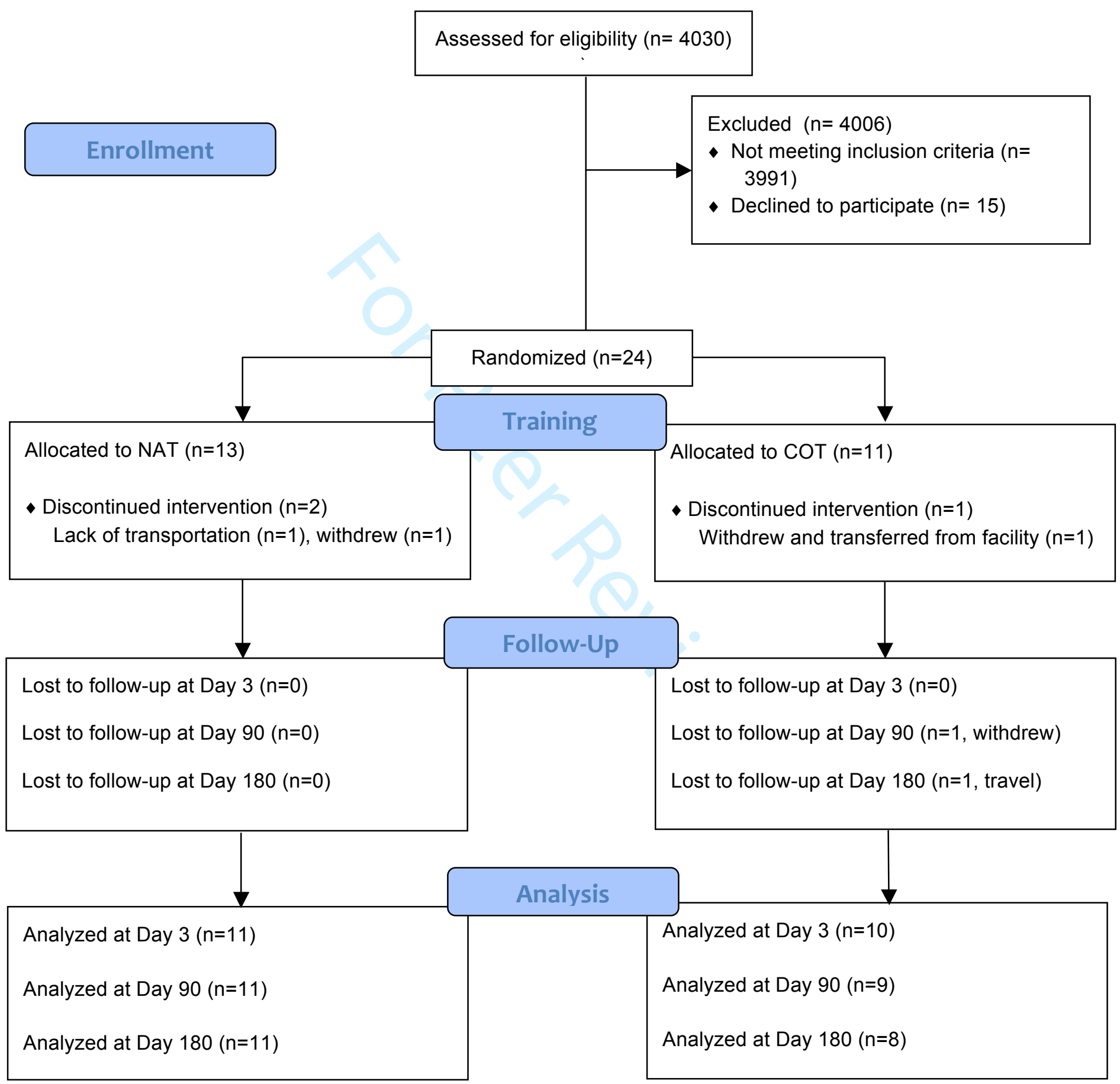




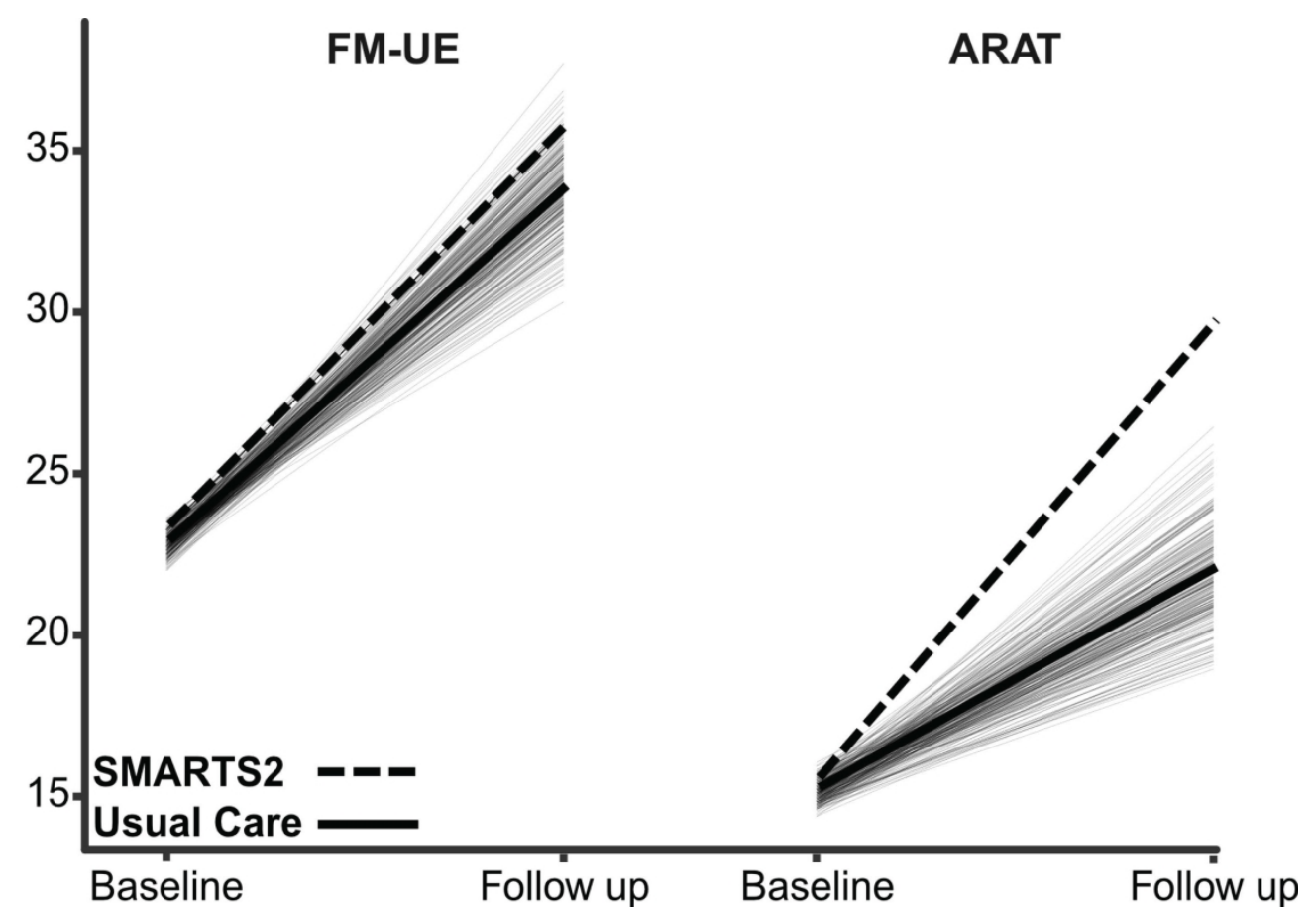

Figure 3. Comparison between the SMARTS2 study cohort receiving intensive therapy (dotted line) and a historical cohort from the EXPLICIT trial receiving usual care (means represented by heavy lines, individual subjects by thin solid lines). The groups were matched for baseline time post-stroke and severity. A significant benefit in upper limb activity and dexterity (ARAT), but not for upper limb impairment (FM-UE), was seen with the intensive therapy provided in SMARTS2.

$480 \times 331 \mathrm{~mm}(150 \times 150 \mathrm{DPI})$ 


\section{Supplemental Material: Comparing a novel neuroanimation experience to conventional therapy for high-dose, intensive upper-limb training in subacute stroke: The SMARTS2 randomized trial}

\section{Extended Methods}

Kinematics of planar reaching

Patients sat at a glass-surface table with their trunk secured to a chair and their hand and forearm immobilized with a splint, allowing only movements of the shoulder and elbow. The forearm was supported using an air-sled system to create a frictionless environment. Patients were instructed to make straight movements with a cursor from a central start circle to four circular targets $(1 \mathrm{~cm}$ radius, $8 \mathrm{~cm}$ distance). Hand position was tracked in real-time using Flock of Birds (Ascension Technology, USA) (JHU, CU) or trakSTAR (Ascension Technology, USA) magnetic recording system at a sampling rate of $130 \mathrm{~Hz}$. Each trial began after the cursor was held inside the start circle for $0.5 \mathrm{~s}$. Audiovisual feedback, a pleasant ding and a change of target color, was provided to movements that ended and remained inside the target with a peak velocity within the range of $20-40 \mathrm{~cm} / \mathrm{s}$. If movements fell outside of this velocity range, auditory feedback was provided indicating whether the movement was too fast or too slow.

Hand position data were analyzed using custom routines in IGOR Pro (Wavemetrics, USA) and $\mathrm{R}^{1}$. Data were low-pass filtered (8 $\mathrm{Hz}$ Butterworth filter) and differentiated to yield tangential velocity and acceleration. Left arm data were spatially flipped across the vertical axis, which allowed for grouping of movements that were directed to targets requiring similar joint configurations. For each movement, we 
identified the velocity peak as the first zero-crossing of acceleration above a velocity threshold of $5 \mathrm{~cm} / \mathrm{s}$. The velocity trace was scanned backwards to identify the time when it crossed $1 \mathrm{~cm} / \mathrm{s}$; this was marked as the movement-start time. Movement-end time was defined as the first point after the peak velocity when the velocity trace remained below $1 \mathrm{~cm} / \mathrm{s}$. The following movements were excluded from further analyses: those that did not reach a peak velocity of $>5 \mathrm{~cm} / \mathrm{s}$, those in which the velocity remained $>1 \mathrm{~cm} / \mathrm{s}$ at the end of the trial, those in which the movement did not leave the starting circle.

The reaching trajectories were analyzed using functional principal component analysis, as previously described (FPCA) ${ }^{2}$. This analysis compares each patient's movements to those of a control group using a global, data-driven metric that is sensitive to changes in overall movement quality. A control group of 12 neurologicallyhealthy volunteers of a similar age distribution as the stroke patients (mean age $=58.4$ years) served as a reference population for kinematic analysis. Subject-specific average squared Mahalanobis distances $\left(\mathrm{AMD}^{2}\right)$ were computed to summarize the distribution of movements for each subject at each target, and then averaged across targets, for each timepoint; larger values of $\mathrm{AMD}^{2}$ indicate movement distributions that are more dissimilar from those of controls.

\section{Finger strength and individuation}

Force transducers (FSG-15N1A, Honeywell; dynamic range 0-50N) measured the downward isometric force exerted at each fingertip with a sampling rate of $200 \mathrm{~Hz}$. The data were digitized using National Instrument USB-621x and MATLAB (MathWorks, 
Natick, MA) Data Acquisition Toolbox. Visual stimuli were presented on a computer monitor running custom routines written in MATLAB Psychophysics Toolbox.

Patients were seated in front of a computer monitor and rested their hands on the keyboards with each finger on top of a key. Ten vertical gray bars representing the 10 fingers were shown at the top of the screen, and another 10 vertical bars below them instructed the amount of force to be exerted. The required force level for each finger per trial was indicated by the position of a horizontal white line.

Maximum voluntary contraction force (MVF) and individuation were tested.

During each MVF trial, patients were asked to press downward with one finger at a time with maximum strength and maintain this force level for 2 seconds. MVF was measured twice for each finger. In the finger individuation trials, patients were instructed to press only one finger at a sub-MVF force level while keeping other fingers immobile. Four target force levels were tested for each finger: $20 \%, 40 \%, 60 \%$, and $80 \%$ of MVF; each level was repeated 4 times. On each trial, the patient was instructed to bring the corresponding horizontal white line up to the force target line and then maintain the required force level for 0.5 seconds. Strength and individuation indices were derived from the raw force traces recorded from the device as described previously ${ }^{3}$.

Strength index. To obtain a measure of finger strength, we used the 95th percentile of the force traces produced across all sampled force data points during the finger-depressing period in each trial and then averaged across the two MVF trials. If the force achieved on one of the two trials was below $60 \%$ of that produced on the other trial, only the larger force was used (13.2\% of trials were excluded). The overall strength of the hand was then calculated by averaging across all five fingers. 
Individuation index. For each trial, we obtained the mean deviation from the baseline force (before GO-cue onset) of each uninstructed finger by averaging over all time bins $(5 \mathrm{~ms} / \mathrm{bin})$ over the entire force trace. A positive linear relationship between the mean deviation of the non-instructed fingers and the instructed finger force is captured by the slope of the regression line of these two variables. To represent the data in a more intuitive manner, we took the negative log of the slope for each of the instructed fingers and averaged across all fingers. A higher value of this index indicates better individuation.

\section{Supplemental References}

1. R: A language and environment for statistical computer [computer program]. Vienna, Austria: R Foundation for Statistical Computing; 2014.

2. Cortes JC, Goldsmith J, Harran MD, et al. A Short and Distinct Time Window for Recovery of Arm Motor Control Early After Stroke Revealed With a Global Measure of Trajectory Kinematics. Neurorehabil Neural Repair. 2017;31(6):552560.

3. $\mathrm{Xu} \mathrm{J}$, Ejaz N, Hertler B, et al. Separable systems for recovery of finger strength and control after stroke. J Neurophysiol. 2017;118(2):1151-1163. 


\section{CONSORT 2010 checklist of information to include when reporting a randomised trial*}

\begin{tabular}{|c|c|c|c|}
\hline Section/Topic & $\begin{array}{l}\text { Item } \\
\text { No }\end{array}$ & Checklist item & $\begin{array}{l}\text { Reported } \\
\text { on page No }\end{array}$ \\
\hline \multicolumn{4}{|l|}{ Title and abstract } \\
\hline & $1 a$ & Identification as a randomised trial in the title & Title \\
\hline & $1 \mathrm{~b}$ & Structured summary of trial design, methods, results, and conclusions (for specific guidance see CONSORT for abstracts) & Abstract \\
\hline \multicolumn{4}{|l|}{ Introduction } \\
\hline \multirow{2}{*}{$\begin{array}{l}\text { Background and } \\
\text { objectives }\end{array}$} & $2 a$ & \multirow{2}{*}{$\begin{array}{l}\text { Scientific background and explanation of rationale } \\
\text { Specific objectives or hypotheses }\end{array}$} & Introduction \\
\hline & $2 b$ & & Introduction \\
\hline \multicolumn{4}{|l|}{ Methods } \\
\hline \multirow[t]{2}{*}{ Trial design } & $3 a$ & \multirow{2}{*}{$\begin{array}{l}\text { Description of trial design (such as parallel, factorial) including allocation ratio } \\
\text { Important changes to methods after trial commencement (such as eligibility criteria), with reasons }\end{array}$} & Methods \\
\hline & $3 b$ & & Methods \\
\hline \multirow[t]{2}{*}{ Participants } & $4 a$ & Eligibility criteria for participants & Methods \\
\hline & $4 \mathrm{~b}$ & Settings and locations where the data were collected & Methods \\
\hline Interventions & 5 & $\begin{array}{l}\text { The interventions for each group with sufficient details to allow replication, including how and when they were } \\
\text { actually administered }\end{array}$ & Methods \\
\hline \multirow[t]{2}{*}{ Outcomes } & $6 a$ & $\begin{array}{l}\text { Completely defined pre-specified primary and secondary outcome measures, including how and when they } \\
\text { were assessed }\end{array}$ & Methods \\
\hline & $6 b$ & Any changes to trial outcomes after the trial commenced, with reasons & Methods \\
\hline \multirow[t]{2}{*}{ Sample size } & $7 a$ & How sample size was determined & $\overline{\text { Methods - stat }}$ \\
\hline & $7 \mathrm{~b}$ & When applicable, explanation of any interim analyses and stopping guidelines & $\mathrm{N} / \mathrm{A}$ \\
\hline \multicolumn{4}{|l|}{ Randomisation: } \\
\hline \multirow{2}{*}{$\begin{array}{l}\text { Sequence } \\
\text { generation }\end{array}$} & $8 a$ & Method used to generate the random allocation sequence & Methods \\
\hline & $8 b$ & Type of randomisation; details of any restriction (such as blocking and block size) & Methods \\
\hline $\begin{array}{l}\text { Allocation } \\
\text { concealment } \\
\text { mechanism }\end{array}$ & 9 & $\begin{array}{l}\text { Mechanism used to implement the random allocation sequence (such as sequentially numbered containers), } \\
\text { describing any steps taken to conceal the sequence until interventions were assigned }\end{array}$ & Methods \\
\hline Implementation & 10 & $\begin{array}{l}\text { Who generated the random allocation sequence, who enrolled participants, and who assigned participants to } \\
\text { interventions }\end{array}$ & Methods \\
\hline Blinding & $11 a$ & If done, who was blinded after assignment to interventions (for example, participants, care providers, those & Methods \\
\hline
\end{tabular}


assessing outcomes) and how

$11 \mathrm{~b}$ If relevant, description of the similarity of interventions

Statistical methods

Statistical methods used to compare groups for primary and secondary outcomes

$12 \mathrm{~b}$ Methods for additional analyses, such as subgroup analyses and adjusted analyses

Methods

Methods

Methods

\section{Results}

Participant flow (a diagram is strongly recommended)

Recruitment

Baseline data

Numbers analysed

Outcomes and estimation

Ancillary analyses

Harms

\section{Discussion}

Limitations

Generalisability

Interpretation

\section{Other information}

Registration

Protocol

Funding
For each group, the numbers of participants who were randomly assigned, received intended treatment, and were analysed for the primary outcome

13b For each group, losses and exclusions after randomisation, together with reasons

14a Dates defining the periods of recruitment and follow-up

14b Why the trial ended or was stopped

15 A table showing baseline demographic and clinical characteristics for each group

16 For each group, number of participants (denominator) included in each analysis and whether the analysis was by original assigned groups

17a For each primary and secondary outcome, results for each group, and the estimated effect size and its precision (such as $95 \%$ confidence interval)

17b For binary outcomes, presentation of both absolute and relative effect sizes is recommended

18 Results of any other analyses performed, including subgroup analyses and adjusted analyses, distinguishing pre-specified from exploratory

19 All important harms or unintended effects in each group (for specific guidance see CONSORT for harms)

20 Trial limitations, addressing sources of potential bias, imprecision, and, if relevant, multiplicity of analyses

21 Generalisability (external validity, applicability) of the trial findings

22 Interpretation consistent with results, balancing benefits and harms, and considering other relevant evidence

23 Registration number and name of trial registry

24 Where the full trial protocol can be accessed, if available

25 Sources of funding and other support (such as supply of drugs), role of funders
Figure 2

Figure 2

Results

Results

Table 1

Figure 2

Table 2

$\mathrm{N} / \mathrm{A}$

Results

Results

Discussion

Discussion

Discussion

Title page

$\mathrm{N} / \mathrm{A}$

Acknowledgments

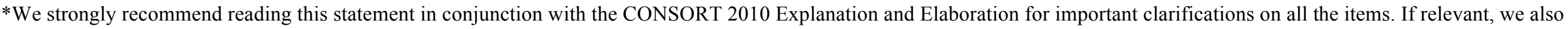

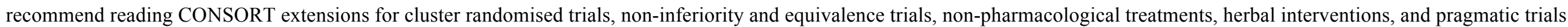
Additional extensions are forthcoming: for those and for up to date references relevant to this checklist, see www.consort-statement.org. 